\title{
A Search for Fast Radio Bursts with the GBNCC Pulsar Survey
}

DOI:

10.3847/1538-4357/aa7d57

\section{Document Version}

Final published version

Link to publication record in Manchester Research Explorer

\section{Citation for published version (APA):}

Chawla, P., Kaspi, V. M., Josephy, A., Rajwade, K. M., Lorimer, D. R., \{Archibald\}, A. M., Decesar, M. E., $\{$ Hessels\}, J. W. T., \{Kaplan\}, D. L., \{Karako-Argaman\}, C., Kondratiev, V. I., Levin, L., Lynch, R. S., McLaughlin, M. A., Ransom, S. M., Roberts, M. S. E., Stairs, I. H., Stovall, K., \{Swiggum\}, J. K., \& van Leeuwen, J. (2017). A Search for Fast Radio Bursts with the GBNCC Pulsar Survey. Astrophysical Journal, 844(2), [140]. https://doi.org/10.3847/1538-4357/aa7d57

\section{Published in:}

Astrophysical Journal

\section{Citing this paper}

Please note that where the full-text provided on Manchester Research Explorer is the Author Accepted Manuscript or Proof version this may differ from the final Published version. If citing, it is advised that you check and use the publisher's definitive version.

\section{General rights}

Copyright and moral rights for the publications made accessible in the Research Explorer are retained by the authors and/or other copyright owners and it is a condition of accessing publications that users recognise and abide by the legal requirements associated with these rights.

\section{Takedown policy}

If you believe that this document breaches copyright please refer to the University of Manchester's Takedown Procedures [http://man.ac.uk/04Y6Bo] or contact uml.scholarlycommunications@manchester.ac.uk providing relevant details, so we can investigate your claim.

\section{OPEN ACCESS}




\title{
A Search for Fast Radio Bursts with the GBNCC Pulsar Survey
}

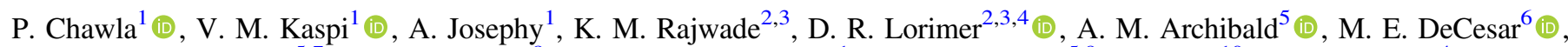 \\ J. W. T. Hessels ${ }^{5,7}$ (1) D. L. Kaplan (1) C. Karako-Argaman ${ }^{1}$, V. I. Kondratiev ${ }^{5,9}$, L. Levin ${ }^{10}$ (1) , R. S. Lynch ${ }^{4}$ (i),

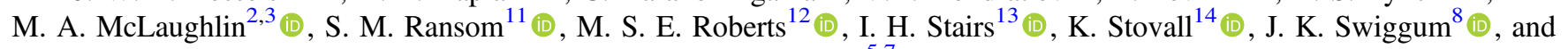 \\ J. van Leeuwen ${ }^{5,7}$ \\ ${ }^{1}$ Department of Physics \& McGill Space Institute, McGill University, 3600 University Street, \\ Montreal, QC H3A 2T8, Canada; pragya.chawla@mail.mcgill.ca \\ ${ }^{2}$ Department of Physics and Astronomy, West Virginia University, Morgantown, WV 26506, USA \\ ${ }^{3}$ Center for Gravitational Waves and Cosmology, West Virginia University, Chestnut Ridge Research Building, Morgantown, WV 26505, USA \\ ${ }^{4}$ Green Bank Observatory, P.O. Box 2, Green Bank, WV 24944, USA \\ ${ }^{5}$ ASTRON, Netherlands Institute for Radio Astronomy, Postbus 2, 7990 AA Dwingeloo, The Netherlands \\ ${ }^{6}$ Department of Physics, Lafayette College, Easton, PA 18042, USA \\ ${ }^{7}$ Anton Pannekoek Institute for Astronomy, University of Amsterdam, Science Park 904, 1098 XH Amsterdam, The Netherlands \\ ${ }^{8}$ Department of Physics, University of Wisconsin-Milwaukee, Milwaukee, WI 53211, USA \\ ${ }_{9}^{9}$ Astro Space Center, Lebedev Physical Institute, Russian Academy of Sciences, Profsoyuznaya str. 84/32, 117997 Moscow, Russia \\ 10 Jodrell Bank Centre for Astrophysics, School of Physics and Astronomy, The University of Manchester, Manchester, M13 9PL, UK \\ ${ }^{11}$ National Radio Astronomy Observatory, 520 Edgemont Road, Charlottesville, VA 22901, USA \\ ${ }^{12}$ New York University, Abu Dhabi, U.A.E. \\ ${ }^{13}$ Department of Physics and Astronomy, University of British Columbia, 6224 Agricultural Road, Vancouver, BC V6T 1Z1, Canada \\ ${ }^{14}$ National Radio Astronomy Observatory, 1003 Lopezville Road, Socorro, NM 87801, USA \\ Received 2017 January 8; revised 2017 June 26; accepted 2017 June 30; published 2017 August 1
}

\begin{abstract}
We report on a search for fast radio bursts (FRBs) with the Green Bank Northern Celestial Cap (GBNCC) Pulsar Survey at $350 \mathrm{MHz}$. Pointings amounting to a total on-sky time of 61 days were searched to a dispersion measure (DM) of $3000 \mathrm{pc} \mathrm{cm}^{-3}$, while the rest (23 days; $29 \%$ of the total time) were searched to a DM of $500 \mathrm{pc} \mathrm{cm}^{-3}$. No FRBs were detected in the pointings observed through 2016 May. We estimate a $95 \%$ confidence upper limit on the FRB rate of $3.6 \times 10^{3} \mathrm{FRBs} \mathrm{Fky}^{-1}$ day $^{-1}$ above a peak flux density of $0.63 \mathrm{Jy}$ at $350 \mathrm{MHz}$ for an intrinsic pulse width of $5 \mathrm{~ms}$. We place constraints on the spectral index $\alpha$ by running simulations for different astrophysical scenarios and cumulative flux density distributions. The nondetection with GBNCC is consistent with the $1.4 \mathrm{GHz}$ rate reported for the Parkes surveys for $\alpha>+0.35$ in the absence of scattering and free-free absorption and $\alpha>-0.3$ in the presence of scattering, for a Euclidean flux distribution. The constraints imply that FRBs exhibit either a flat spectrum or a spectral turnover at frequencies above $400 \mathrm{MHz}$. These constraints also allow estimation of the number of bursts that can be detected with current and upcoming surveys. We predict that CHIME may detect anywhere from several to $\sim 50$ FRBs per day (depending on model assumptions), making it well suited for interesting constraints on spectral index, the $\log N-\log S$ slope, and pulse profile evolution across its bandwidth (400-800 MHz).
\end{abstract}

Key words: methods: data analysis - methods: statistical - pulsars: general - surveys

\section{Introduction}

Fast radio bursts (FRBs) are bright, millisecond-duration events occurring in the radio sky. Their origin is still unknown. At the time of writing, eighteen FRBs have been discovered (Lorimer et al. 2007; Keane et al. 2012; Thornton et al. 2013; Burke-Spolaor \& Bannister 2014; Masui et al. 2015; Petroff et al. 2015; Ravi et al. 2015, 2016; Champion et al. 2016; Keane et al. 2016), with only one source (Spitler et al. 2014, 2016) known to repeat. A catalog of these bursts and their properties is made available by Petroff et al. (2016). ${ }^{15}$ These transient events can be distinguished from pulsars and rotating radio transients (RRATs) on the basis of their dispersion measure (DM), which is a measure of the integrated free electron density along the line of sight in the intervening medium. The bursts have DMs that are 1.4-35 times the maximum predicted along the line of sight by the NE2001 model of electron density in our Galaxy (Cordes \& Lazio 2002).

\footnotetext{
15 http://www.astronomy.swin.edu.au/pulsar/frbcat/
}

The dominant contribution to the excess DM of FRBs can arise from the intergalactic medium (IGM), the host galaxy of the FRB progenitor, or possibly from a high electron density, compact structure in our Galaxy. The interferometric localization of bursts from the repeating FRB121102 provides evidence of its association with an optical counterpart (Chatterjee et al. 2017). Spectroscopic follow-up by Tendulkar et al. (2017) confirms the optical counterpart as being the host galaxy of the FRB and characterizes it as a low-metallicity, star-forming dwarf galaxy located at a redshift of $z=0.19273$ (8). The observations of Masui et al. (2015) also support an extragalactic origin with scattering and scintillation in FRB110523, suggesting that the majority of the scattering originates from within the typical size scale of a galaxy. These observations lend support to models with extragalactic progenitors of FRBs such as giant pulses from extragalactic neutron stars (Cordes \& Wasserman 2016) and magnetar giant flares (Popov \& Postnov 2013; Kulkarni et al. 2015). Interferometric localizations of more FRBs are essential to conclusively determine the source of the excess DM and the nature of the FRB progenitors for the broader FRB population. 
All but one known FRB (Masui et al. 2015) has been detected at frequencies greater than $1 \mathrm{GHz}$. Detection or stringent limits at lower frequencies are crucial for understanding properties of FRBs such as their spectral index and pulse profile evolution with frequency. Searches at low frequencies with telescopes such as LOFAR (Coenen et al. 2014; Karastergiou et al. 2015), Arecibo (Deneva et al. 2016), and MWA (Tingay et al. 2015; Rowlinson et al. 2016) have so far not resulted in any detections. Deneva et al. (2016) report an upper limit on the FRB rate at $327 \mathrm{MHz}$ of $10^{5}$ FRBs sky $^{-1}$ day $^{-1}$ for a flux density threshold of $83 \mathrm{mJy}$ and pulse width of $10 \mathrm{~ms}$. A nondetection with the LOFAR Pilot Pulsar Survey at $142 \mathrm{MHz}$ allowed Coenen et al. (2014) to place an upper limit of $150 \mathrm{FRBs} \mathrm{sky}^{-1} \mathrm{day}^{-1}$, for bursts brighter than $107 \mathrm{Jy}$ at burst duration $0.66 \mathrm{~ms}$. Karastergiou et al. (2015) report an upper limit of $29 \mathrm{FRBs}^{\mathrm{sky}}{ }^{-1} \mathrm{day}^{-1}$ for bursts with flux density above $62 \mathrm{Jy}$ at $145 \mathrm{MHz}$ and a pulse width of $5 \mathrm{~ms}$, based on observations with the UK station of the LOFAR radio telescope. The upper limits on the FRB rate reported thus far from these low-frequency radio surveys are not particularly constraining because of limitations in total observing time and volume searched. With observations to date amounting to a total on-sky time of 84 days, the Green Bank Northern Celestial Cap (GBNCC) Pulsar Survey (Stovall et al. 2014) can provide the strongest constraints yet on the FRB rate and spectral index in the frequency range of $300-400 \mathrm{MHz}$.

The GBNCC survey is also important for predicting the FRB yield of upcoming low-frequency telescopes such as the Canadian Hydrogen Intensity Mapping Experiment (CHIME). With its large field of view and good sensitivity, CHIME is predicted to discover tens of FRBs per day (Connor et al. 2016; Rajwade \& Lorimer 2017) in its frequency range of $400-800 \mathrm{MHz}$. The GBNCC survey is thus well placed to determine the expected detection rate for the lower part of the CHIME band.

In this paper, we present results from the search for FRBs in GBNCC survey pointings observed through 2016 May. For the purpose of our search and subsequent analysis, we define an FRB as an astrophysical pulse with a DM greater than twice the maximum line-of-sight Galactic DM. The suggestion by Bannister \& Madsen (2014) of a possibly Galactic origin of the excess DM of the only FRB with a DM ratio $<2$, FRB010621 (Keane et al. 2012), lends support to our choice of a DM ratio of 2 for the FRB definition.

Our paper is organized as follows. In Section 2, we give a description of the survey and its sensitivity. We describe the data analysis pipeline in Section 3 and place constraints on the FRB rate in Section 4. In Section 5, we constrain the mean spectral index of FRBs by performing Monte Carlo simulations of a population of FRBs. We discuss the implications for current and upcoming surveys in Section 6 and present our summary and conclusions in Section 7.

\section{Observations}

\subsection{Survey Description}

The Green Bank Northern Celestial Cap (GBNCC) Pulsar Survey (Stovall et al. 2014) began in 2009 with the aim of searching for pulsars and RRATs, particularly millisecond pulsars suitable for inclusion in the North American Nanohertz Observatory for Gravitational Waves (NANOGrav) pulsar timing array. ${ }^{16}$ The search is conducted using the $100 \mathrm{~m}$

\footnotetext{
16 http://nanograv.org
}

diameter Robert C. Byrd Green Bank Telescope (GBT) at a frequency of $350 \mathrm{MHz}$. Data spanning $100 \mathrm{MHz}$ of bandwidth split into 4096 frequency channels are recorded with the Green Bank Ultimate Pulsar Processing Instrument (GUPPI). Each pointing on the sky is observed for $120 \mathrm{~s}$ and sampled with an $81.92 \mu$ s time resolution.

The entire sky visible to the GBT $\left(\delta>-40^{\circ}\right)$ has been divided into $\sim 125,000$ pointings, around 75,000 of which have been observed through 2016 May. In the initial days of the survey, data were searched to a maximum DM of $500 \mathrm{pc} \mathrm{cm}^{-3}$. Motivated by the discovery of FRBs, the maximum DM for the search was increased to $3000 \mathrm{pc} \mathrm{cm}^{-3}$. However, the initial pointings are yet to be reprocessed with this updated parameter. A total of $71 \%$ of the pointings were searched to a DM of $3000 \mathrm{pc} \mathrm{cm}^{-3}$, and $29 \%$ of the pointings were searched to a $\mathrm{DM}$ of $500 \mathrm{pc} \mathrm{cm}^{-3}$. The search in DM space is conducted by stepping over a range of trial DMs, with $\triangle \mathrm{DM}$ being the step size between consecutive trials. The DM step sizes used by the search pipeline for the GBNCC survey are mentioned in the caption to Figure 3.

Not all pointings observed by the GBNCC survey were examined during the analysis reported on here. Pointings searched to a DM of $500 \mathrm{pc} \mathrm{cm}^{-3}$ for which the maximum line-of-sight Galactic DM predicted by the NE2001 model (Cordes \& Lazio 2002) was greater than $100 \mathrm{pc} \mathrm{cm}^{-3}$ were not inspected. This is because our adopted definition of an FRB implies that these 7000 pointings searched over an extremely small range of extragalactic DMs as compared to the rest of the pointings. Removal of an additional 3000 pointings that were rendered unusable by the presence of radio frequency interference (RFI) limited the total observing time for the FRB search to 84 days. The time corresponding to an estimated masking fraction of $2 \%$ for all pointings has been subtracted from the total time on sky reported here.

Figure 1 shows the GBNCC pointings included in our FRB search overlaid on the sky map of the maximum Galactic DM predicted by the NE2001 model (Cordes \& Lazio 2002). The temporal distribution of the pointings is shown in Figure 2.

\subsection{Survey Sensitivity}

The minimum detectable flux density $S_{\min }$ for FRBs searched with the GBNCC survey can be calculated using the expression derived by Cordes \& McLaughlin (2003):

$$
S_{\min }=\frac{\beta(\mathrm{S} / \mathrm{N})_{\mathrm{b}}\left(T_{\mathrm{rec}}+T_{\mathrm{sky}}\right)}{G W_{\mathrm{i}}} \sqrt{\frac{W_{\mathrm{b}}}{n_{\mathrm{p}} \Delta \nu}},
$$

where $\beta$ is a factor accounting for digitization losses, $(\mathrm{S} / \mathrm{N})_{\mathrm{b}}$ is the minimum detectable signal-to-noise ratio of the broadened pulse, $T_{\text {rec }}$ is the receiver temperature, $T_{\text {sky }}$ is the sky temperature, $W_{\mathrm{i}}$ and $W_{\mathrm{b}}$ are the intrinsic and broadened pulse widths, respectively, $G$ is the telescope gain, $n_{\mathrm{p}}$ is the number of polarizations summed, and $\Delta \nu$ is the bandwidth. Values of the above-mentioned parameters for the GBNCC survey are listed in Table 1 . We use $\Delta \nu=75 \mathrm{MHz}$ instead of the recorded bandwidth of $100 \mathrm{MHz}$ to account for roll-off at the bandpass edges and for the estimated masking fraction of $5 \%$ in the frequency domain. The average sky temperature at $350 \mathrm{MHz}$, $T_{\text {sky }}=44 \mathrm{~K}$, along the line of sight for all the pointings included in the FRB search has been estimated using the 


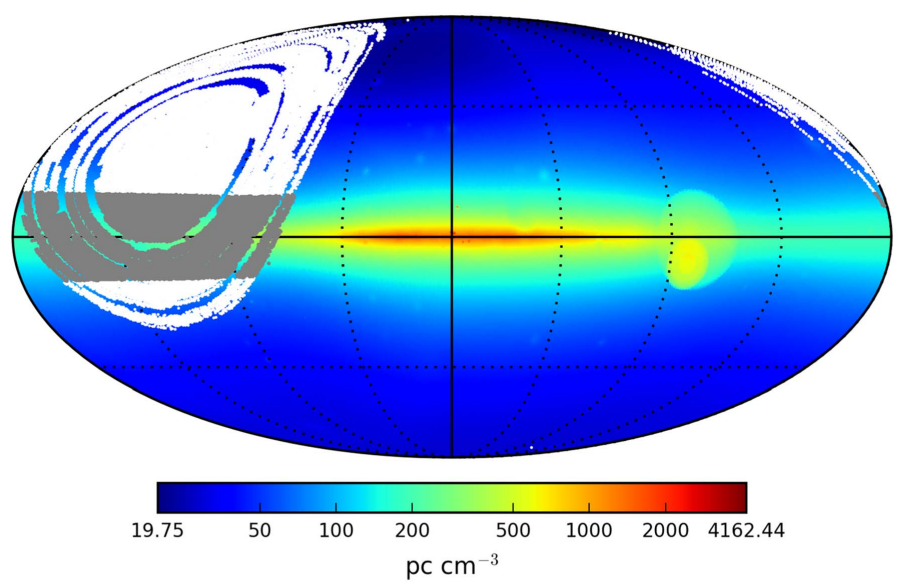

(a)

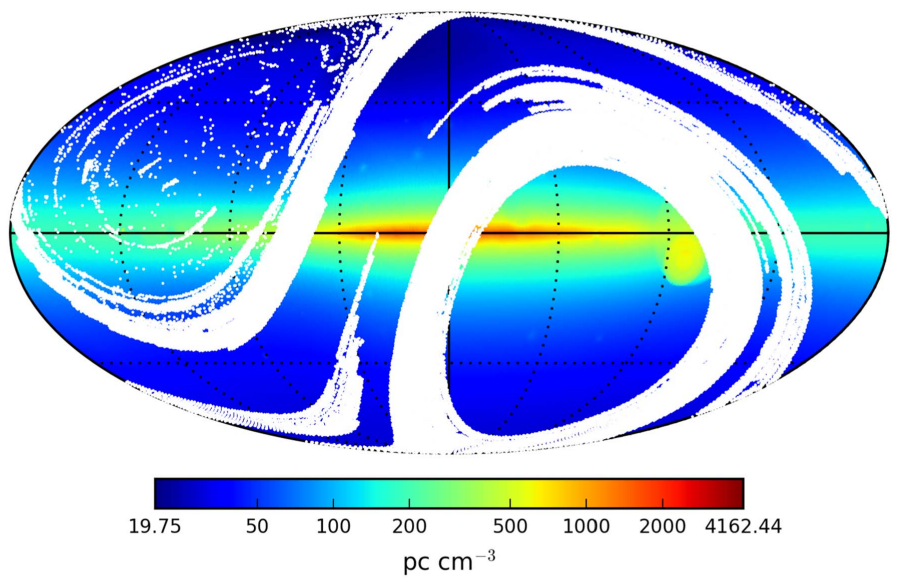

(b)

Figure 1. Full-sky map in Galactic coordinates with GBNCC pointings (marked in white) overlaid on the maximum Galactic DM predicted by the NE2001 model. Panel (a) shows the pointings searched to a DM of $500 \mathrm{pc} \mathrm{cm}^{-3}$, with the excluded pointings having a predicted maximum DM $>100 \mathrm{pc} \mathrm{cm}$ marked in gray, and panel (b) shows the pointings searched to a DM of $3000 \mathrm{pc} \mathrm{cm}^{-3}$. Pointings rendered unusable by the presence of RFI have not been plotted here.

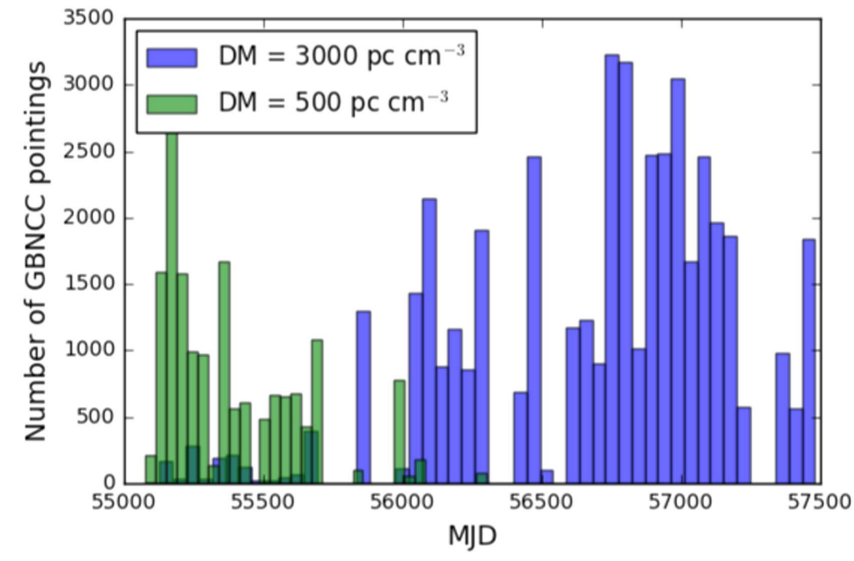

Figure 2. Temporal distribution of GBNCC pointings searched for FRBs. The $\mathrm{x}$-axis denotes the Modified Julian Date (MJD) at the start of the observation. The pointings marked in green have been searched to a DM of $500 \mathrm{pc} \mathrm{cm}^{-3}$, and those in blue have been searched to a DM of $3000 \mathrm{pc} \mathrm{cm}^{-3}$.

$408 \mathrm{MHz}$ all-sky map (Remazeilles et al. 2015) and a spectral index of -2.6 for Galactic emission.

The broadened pulse width $W_{\mathrm{b}}$ accounts for both instrumental and propagation effects and is computed from the quadrature sum as follows:

$$
W_{\mathrm{b}}=\sqrt{W_{\mathrm{i}}^{2}+t_{\mathrm{samp}}^{2}+t_{\mathrm{chan}}^{2}+t_{\mathrm{scatt}}^{2}} .
$$

Here $t_{\text {samp }}$ is the sampling time and $t_{\text {scatt }}$ is the scattering time arising from multipath propagation of signals caused by an ionized medium. The dispersive delay within each frequency channel, $t_{\text {chan }}$, is calculated (see, e.g., Lorimer \& Kramer 2005) as follows:

$$
t_{\text {chan }}=8.3 \mu s\left(\frac{\Delta \nu_{\text {chan }}}{\mathrm{MHz}}\right)\left(\frac{\nu}{\mathrm{GHz}}\right)^{-3}\left(\frac{\mathrm{DM}}{\mathrm{pc} \mathrm{cm}^{-3}}\right)
$$

where $\nu$ is the central observing frequency and $\Delta \nu_{\text {chan }}$ is the channel bandwidth.
For an intrinsic pulse width $W_{\mathrm{i}}=5 \mathrm{~ms}$, scattering time $t_{\text {scatt }}=0 \mathrm{~ms}$, and a DM of $756 \mathrm{pc} \mathrm{cm}^{-3}$ (mean DM of known FRBs; Petroff et al. 2016), the minimum detectable flux density for the GBNCC survey is $0.63 \mathrm{Jy}$. We note that there is a reduction in sensitivity to high-DM events since the dispersive delay for these events across a bandwidth of $100 \mathrm{MHz}$ is a large fraction of the observation time per pointing. However, a significant fraction $(29 \%)$ of our pointings have been searched to a DM of $500 \mathrm{pc} \mathrm{cm}^{-3}$, where this effect is not important. Also, since the highest DM observed for a known FRB is $1629 \mathrm{pc} \mathrm{cm}^{-3}$ (Champion et al. 2016), the sensitivity is impacted only for a small region of the parameter space.

The minimum detectable flux density is plotted as a function of intrinsic pulse width and scattering time, for different DM step sizes, in Figure 3. The minimum detectable $\mathrm{S} / \mathrm{N}$, used for the calculation of the minimum detectable flux density, is also dependent on the intrinsic pulse width, scattering timescale, and DM step size. The dependence of the $\mathrm{S} / \mathrm{N}$ on these variables is part of the code used to search and rank FRB candidates, RRATtrap (described in Section 3.1). The rationale for this dependence is detailed in Section 3.1.1.

\section{Analysis}

The analysis pipeline, based on the PRESTO software package (Ransom 2001), ${ }^{17}$ is run on the Guillimin High Performance Computing (HPC) cluster operated at McGill University by CLUMEQ and Compute Canada. The first step of processing involves searching for and masking time samples and frequency channels containing RFI. The effect of dispersion is then corrected for by dedispersing the data at a large number of trial DMs up to a maximum of $500 \mathrm{pc} \mathrm{cm}^{-3}$ or $3000 \mathrm{pc} \mathrm{cm}^{-3}$. The dedispersed and downsampled time series for each trial DM is subsequently searched for single pulses using a matched filtering algorithm that convolves the time series with boxcars having widths ranging from $81.92 \mu$ s to $100 \mathrm{~ms}$. All single-pulse events with $\mathrm{S} / \mathrm{N}$ greater than 5 are stored for further processing. The above-mentioned analysis has been described in detail in Stovall et al. (2014).

\footnotetext{
$\overline{17 \text { http://www.cv.nrao.edu/ sransom/presto }}$
} 
Table 1

Search Parameters for Various FRB Surveys

\begin{tabular}{|c|c|c|c|c|c|c|c|c|}
\hline Survey & $\begin{array}{l}\text { Field of View } \\
\left(\mathrm{deg}^{2}\right)\end{array}$ & $\begin{array}{l}\text { Bandwidth } \\
\text { (MHz) }\end{array}$ & $\begin{array}{l}\text { Center Freq. } \\
(\mathrm{MHz})\end{array}$ & $\begin{array}{l}\text { No. of Freq. } \\
\text { Channels }\end{array}$ & $\begin{array}{l}\text { Polarizations } \\
\text { Summed }\end{array}$ & $\begin{array}{l}\text { Gain }^{\mathrm{a}} \\
(\mathrm{K} / \mathrm{Jy})\end{array}$ & $\begin{array}{l}T_{\text {rec }} \\
(\mathrm{K})\end{array}$ & References \\
\hline GBNCC & 0.408 & 100 & 350 & 4096 & 2 & 2 & 23 & 1 \\
\hline PARKES $^{\mathrm{b}}$ & $0.559^{\mathrm{c}}$ & 340 & 1352 & 1024 & 2 & 0.64 & 23 & 2 \\
\hline UTMOST & $4.64 \times 2.14$ & 31.25 & 843 & 40 & 1 & 3.6 & 70 & 3 \\
\hline PALFA & 0.022 & 322 & 1375 & 960 & 2 & \multicolumn{2}{|c|}{$\mathrm{SEFD}=5$} & 4 \\
\hline CHIME & 134 & 400 & 600 & 16000 & 2 & 1.38 & 50 & 5 \\
\hline AO327 & 0.049 & 57 & 327 & 1024 & 2 & 11 & 115 & 6 \\
\hline ARTEMIS (LOFAR) & 24 & 6 & 145 & 64 & 2 & \multicolumn{2}{|c|}{$\mathrm{SEFD}=1100$} & 8 \\
\hline ALERT (APERTIF) & 8.7 & 300 & 1400 & 1024 & 2 & 0.96 & 75 & 9 \\
\hline V-FASTR & 0.364 & $32^{\mathrm{d}}$ & 1550 & $512^{\mathrm{d}}$ & 2 & \multicolumn{2}{|c|}{$\mathrm{SEFD}=311$} & 10,11 \\
\hline \multirow[t]{2}{*}{ MWA } & 600 & 30.72 & 155 & 24 & 2 & $1^{\mathrm{e}}$ & 50 & 12 \\
\hline & 145 & 30.72 & 182 & $\cdots$ & 2 & $1^{\mathrm{e}}$ & 50 & 13 \\
\hline VLA & 0.283 & 256 & 1396 & 256 & 2 & \multicolumn{2}{|c|}{$\mathrm{SEFD}=16$} & 14 \\
\hline
\end{tabular}

Notes.

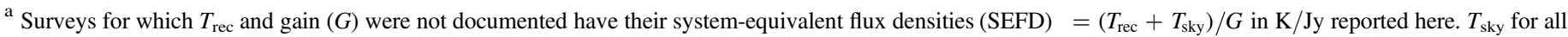
other surveys has been evaluated assuming an average sky temperature of $34 \mathrm{~K}$ at $408 \mathrm{MHz}$ and a spectral index of -2.6 (Haslam et al. 1982).

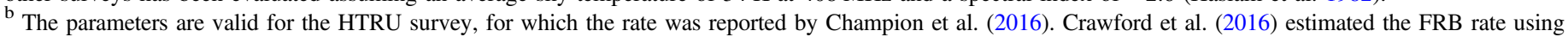
several Parkes surveys, the parameters for which have been reported in their paper.

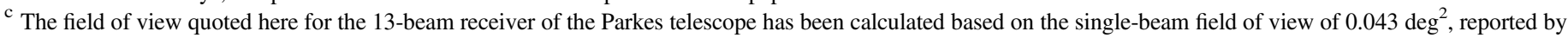
Burke-Spolaor \& Bannister (2014).

$\mathrm{d}$ The number of frequency channels and bandwidth reported for V-FASTR are representative values, as the observing setup can vary between observations.

e The gain for MWA is given by $A_{\text {eff }} / 2 k$, where $k$ is the Boltzmann constant and $A_{\text {eff }}$ is the effective area of the telescope reported by Tingay et al. (2013).

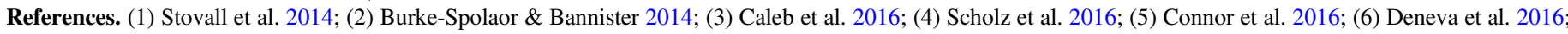

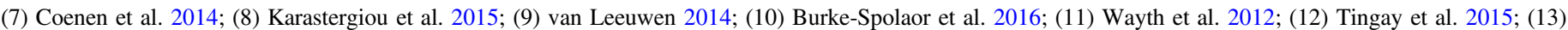
Rowlinson et al. 2016; (14) Law et al. 2015.

The single-pulse output is processed by a grouping and rating algorithm RRATtrap, ${ }^{18}$ which has aided in the discovery of 10 RRATs in GBNCC survey data (Karako-Argaman et al. 2015).

\subsection{RRATtrap}

The large number of DM trials ensures that each pulse (astrophysical or RFI) is detected as multiple single-pulse events that are closely spaced in DM and time. RRATtrap groups all such events and ranks the groups based on how closely they match the behavior of an astrophysical pulse. It then produces colorized DM versus time plots for several DM ranges with groups of different ranks plotted in different colors.

A group of fewer than 30 single-pulse events occurring within a fixed DM and time threshold is classified as noise and not processed further. A considerable fraction of the singlepulse events in our pointings fall in this category. Strong narrowband RFI is another major source of single-pulse events. The algorithm deals with these signals, which we know have a terrestrial origin, by assigning a low rank to groups with the $\mathrm{S} / \mathrm{N}$ peaking at a $\mathrm{DM}<2 \mathrm{pc} \mathrm{cm}^{-3}$. A low rank is also assigned to a group corresponding to a narrowband signal, identified by it being detected with a constant $\mathrm{S} / \mathrm{N}$ over a large range of DMs. A bright, broadband signal from an astrophysical source will be detected with the maximum $\mathrm{S} / \mathrm{N}$ at an optimal DM and with lower $\mathrm{S} / \mathrm{N}$ at closely spaced trial DMs above or below the optimal DM, due to dispersive smearing.

\footnotetext{
${ }^{18}$ The code is available at https://github.com/ajosephy/Clustering/ and is a modified version of the code by Karako-Argaman et al. (2015), which is available at https://github.com/ckarako/RRATtrap.
}

Groups exhibiting this characteristic of astrophysical pulses are ranked highly.

\subsubsection{RRATtrap Sensitivity}

RRATtrap exhibits a significant variation in sensitivity with pulse width, due to our requirement of a minimum of 30 singlepulse events for a group to be ranked. Sensitivity to extremely narrow pulses is reduced since dispersive smearing prevents the detection of the pulse at $30 \mathrm{DM}$ trials. The reduction in the sensitivity is maximum at high DMs, where the DM step size increases to $0.5 \mathrm{pc} \mathrm{cm}^{-3}$.

In order to determine whether a pulse will be ranked by RRATtrap, we first obtain the peak flux $S$ corresponding to the $\mathrm{S} / \mathrm{N}$ of the pulse at the optimal DM $\left(\mathrm{S} / \mathrm{N}_{\text {peak }}\right)$, using Equation (1). The reduction in the peak flux $S$ of the pulse due to dedispersion at an incorrect trial DM is modeled by the following equation derived by Cordes \& McLaughlin (2003):

$$
\frac{S(\delta \mathrm{DM})}{S}=\frac{\sqrt{\pi}}{2} \zeta^{-1} \operatorname{erf} \zeta
$$

where

$$
\zeta=6.91 \times 10^{-3} \delta \mathrm{DM} \frac{\Delta \nu}{W_{\mathrm{i}, \mathrm{ms}} \nu_{\mathrm{GHz}}^{3}} .
$$

Here $\nu_{\mathrm{GHz}}=0.350 \mathrm{GHz}$ is the center frequency of the GBNCC survey and $S(\delta \mathrm{DM})$ is the reduced flux measured at a trial DM differing from the optimal DM by $\delta \mathrm{DM}$. The width of the pulse dedispersed at an incorrect trial DM is given by $W(\delta \mathrm{DM})=S W_{\mathrm{i}} / S(\delta \mathrm{DM})$ since dispersive smearing conserves pulse area $A=S W_{\mathrm{i}}$ (Cordes \& McLaughlin 2003). A 


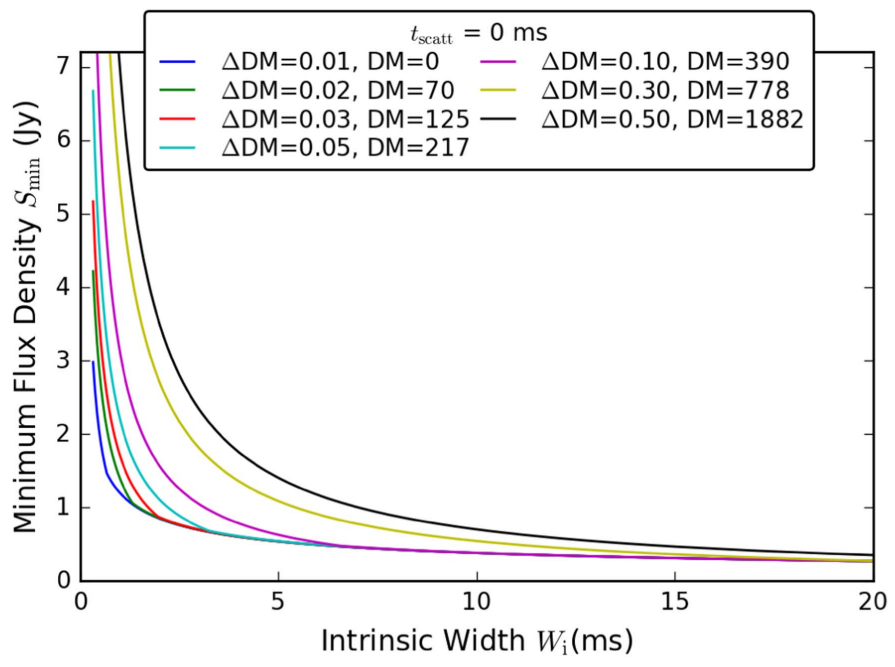

(a)

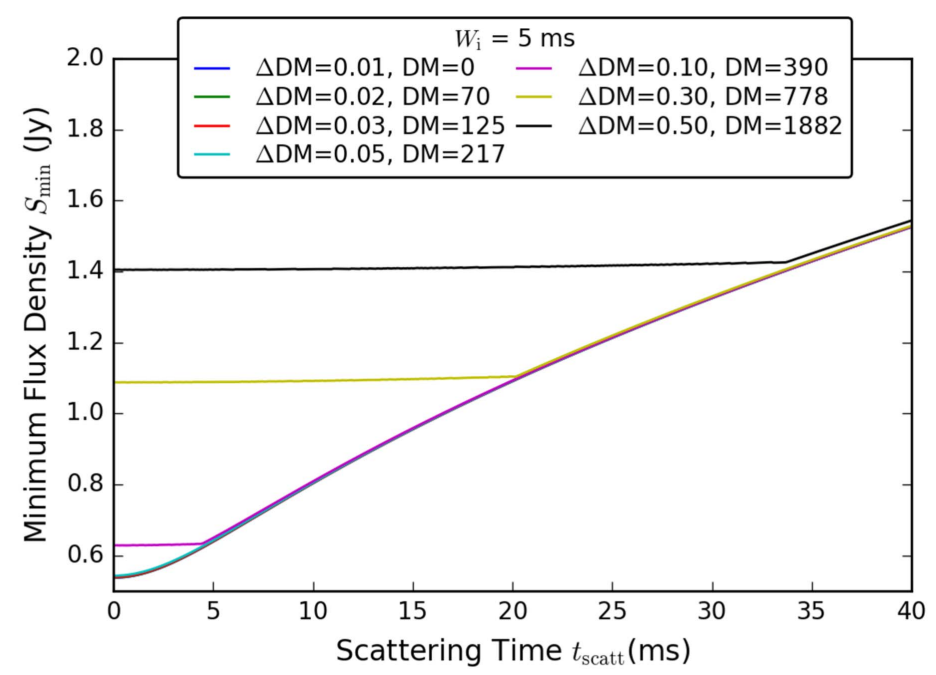

(b)

Figure 3. Minimum detectable flux density $S_{\min }$, corresponding to DM step sizes ( $\Delta \mathrm{DM}$ ) of $0.01,0.02,0.03,0.05,0.10,0.30$, and $0.50 \mathrm{pc} \mathrm{cm}^{-3}$, plotted as a function of intrinsic pulse width $W_{\mathrm{i}}$ in panel (a) and scattering timescale $t_{\text {scatt }}$ at $350 \mathrm{MHz}$ in panel (b). The $\mathrm{S} / \mathrm{N}$ used in the estimation of the minimum detectable flux density is a function of the pulse width $W_{\mathrm{i}}$, scattering timescale $t_{\text {scatt }}$, and the DM step size, $\Delta \mathrm{DM}$. The specified DM values have units of pc $\mathrm{cm}^{-3}$ and are the lower bounds of the trial DM ranges for the given DM step sizes.

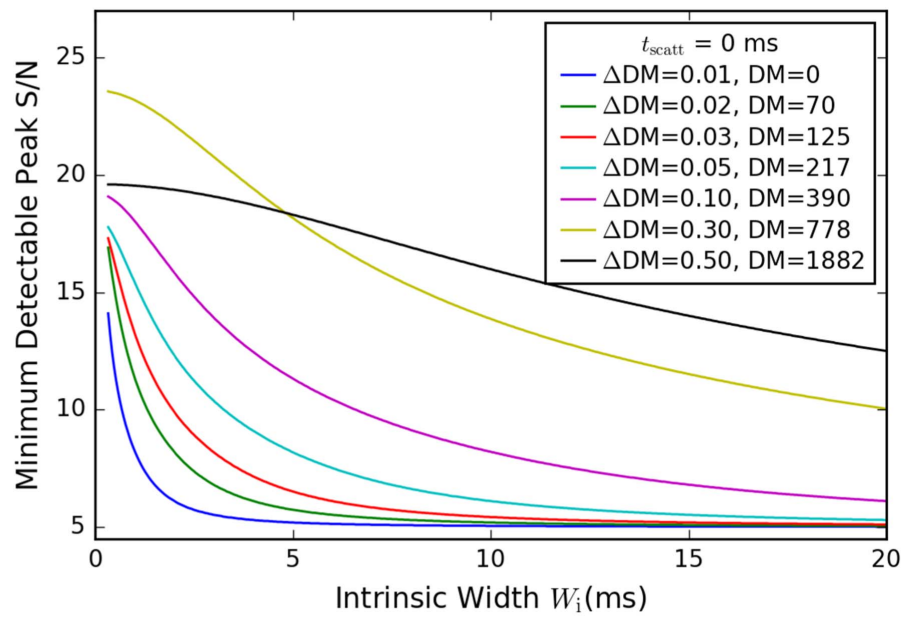

(a)

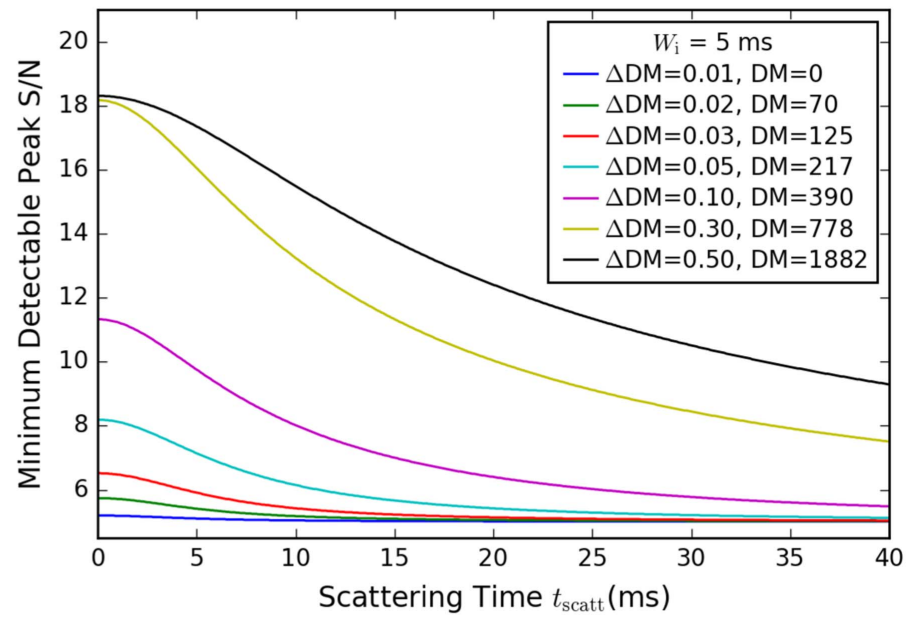

(b)

Figure 4. Minimum detectable peak $\mathrm{S} / \mathrm{N}$ with RRATtrap, corresponding to DM step sizes $(\Delta \mathrm{DM})$ of $0.01,0.02,0.03,0.05,0.10,0.30$, and $0.50 \mathrm{pc} \mathrm{cm}^{-3}$, plotted as a function of intrinsic pulse width $W_{\mathrm{i}}$ in panel (a) and scattering timescale $t_{\text {scatt }}$ at $350 \mathrm{MHz}$ in panel (b). The specified DM values have units of pc cm ${ }^{-3}$ and are the lower bounds of the trial DM ranges for the given DM step sizes.

single-pulse event at a trial DM, with a DM error of $\delta \mathrm{DM}$, will therefore be detected with an $\mathrm{S} / \mathrm{N}$ that can be determined by substituting the reduced flux $S(\delta \mathrm{DM})$, the intrinsic pulse width $W(\delta \mathrm{DM})$, and other parameters of the GBNCC survey in Equation (1).

For a given pulse width, we can thus obtain the minimum value of the peak $\mathrm{S} / \mathrm{N}$ that will allow detection of 30 singlepulse events with an $\mathrm{S} / \mathrm{N}>5$. The minimum detectable peak $\mathrm{S} / \mathrm{N}$ is plotted as a function of intrinsic pulse width and scattering time, for different DM step sizes, in Figure 4. The $\mathrm{S} / \mathrm{N}$ used to calculate the threshold flux density of the GBNCC survey is set to be the minimum value of the peak $\mathrm{S} / \mathrm{N}$ evaluated using the above-mentioned method or 10, whichever is greater. This is done to account for the fact that only pointings having an FRB candidate with an $\mathrm{S} / \mathrm{N}>10$ were visually inspected (see Section 3.2).

\subsubsection{Modifications to RRATtrap}

Algorithmic changes were made to the grouping stage. Initially, this was done via "agglomerative hierarchical clustering" (AHC; Anderberg 1973), which runs in $\mathcal{O}\left(n^{3}\right)$ time for the simplest implementation, where $n$ is the number of single-pulse events. AHC is a bottom-up approach where all events are first initialized as individual groups and then iteratively merged based on proximity in DM and time. Merging terminates once the minimum separation between groups, in either DM or time, is above some dimension-specific threshold. The threshold in time is taken as $100 \mathrm{~ms}$, corresponding to the largest boxcar used to detect pulses. The threshold in DM is taken as $0.5 \mathrm{pc} \mathrm{cm}^{-3}$ and is increased for large DMs, where the separation in trial DMs increases.

The AHC method was replaced with the "density-based spatial clustering of applications with noise" (DBSCAN) 
algorithm (Ester et al. 1996), which runs in $\mathcal{O}(n \log n)$ time. DBSCAN works by taking an arbitrary event and running a nearest-neighbor query to start a group including events that are sufficiently nearby. This group is then iteratively grown outward by repeating the neighborhood query for newly added members. Once the reachable events are exhausted, the group is complete and the process repeats for the next unvisited event.

Since the distance thresholds used by both algorithms determine whether or not two events belong to the same group, identical thresholds yield identical output. The purpose of the change was to reduce runtime. The performance improvement is largely due to storing the single-pulse events in a $k$-d tree (Bentley 1975), which allows neighborhood queries to be done in logarithmic time.

A $k$-d tree is a space-partitioning data structure used to organize data existing in $k$ dimensions. For our two dimensions, the tree is constructed as follows. The median event in time is taken as the root, which partitions the plane in two. Now for each side, median events in DM are taken to further partition the plane into four regions-these two events are the nodes in the second level of the tree. This process continues, cycling in DM and time, until all events exist as nodes on the tree. The construction of the tree takes $\mathcal{O}(n \log n)$ time.

\subsection{Visual Inspection}

A total of $72 \%$ of the pointings had at least one single pulse with an $\mathrm{S} / \mathrm{N}>10$. These 44,000 pointings were processed with the modified version of RRATtrap to group and rank single-pulse events at a DM greater than twice the maximum line-of-sight Galactic DM, $\mathrm{DM}_{\mathrm{Mw}}$. There is a $10 \%$ chance that an astrophysical pulse will not be ranked highly by RRATtrap (Karako-Argaman et al. 2015). To ensure no effect of this false negative rate on our search, we did not apply RRATtrap ranks as a criterion for visual inspection and inspected plots (corresponding to $\mathrm{DM}$ ranges for which $\mathrm{DM}>2 \mathrm{DM}_{\mathrm{MW}}$ ) for all 44,000 pointings, regardless of the ranks of the groups they contained. However, the colors corresponding to the ranks guided the eye during the inspection of the plots. We flagged potential astrophysical candidates in these pointings and obtained their dynamic spectrum, or their intensity as a function of frequency and time. All flagged candidates had characteristics consistent with RFI and showed no evidence of a dispersive sweep. We conclude that no FRB with an $\mathrm{S} / \mathrm{N}$ greater than the detection threshold of RRATtrap (see Figure 4) was present in these pointings.

\section{Calculation of FRB Rate}

\subsection{Estimation of Sky Rate}

The nondetection of FRBs in our search is a significant result since it constrains the all-sky FRB rate at $350 \mathrm{MHz}$. Assuming that FRBs follow Poisson statistics, the probability of detecting $N$ FRBs is

$$
P(N)=\frac{(R T \Omega)^{N} e^{-(R T \Omega)}}{N !},
$$

where $\Omega$ is the solid angle of the beam, $T$ is the total observing time, and $R$ is the FRB rate per unit solid angle. The $95 \%$ confidence upper limit on the rate is the upper bound for which normalization and integration of Equation (6), with a lower bound of $R=0$, yield a value of 0.95 for the case $N=0$.
We will be reporting the rate for two different beam areas, one for the field of view corresponding to the FWHM of the GBT beam and another for the field of view at the edge of which the gain is equal to $0.64 \mathrm{~K} / \mathrm{Jy}$ (i.e., the Parkes $1.4 \mathrm{GHz}$ on-axis gain; Burke-Spolaor \& Bannister 2014). The former will be referred to as the FWHM case, and the latter as the Parkes-equivalent case. Since all but two of the currently known FRBs have been detected using the Parkes telescope, we estimate the rate for the Parkes-equivalent case to facilitate comparison with the Parkes $1.4 \mathrm{GHz}$ rate estimate (Champion et al. 2016; Crawford et al. 2016). Knowing that the GBT beam is well approximated by a 2D symmetric Gaussian, we obtain $\Omega=0.408 \mathrm{deg}^{2}$ for the FWHM case $\left(\theta_{0}=36^{\prime}\right)^{19}$ and $\Omega=0.672 \operatorname{deg}^{2}\left(\theta_{0}=46^{\prime}\right)$ for the Parkes-equivalent case.

The total time on sky, $T$, is 61 days for GBNCC pointings searched to a DM of $3000 \mathrm{pc} \mathrm{cm}^{-3}$ and 23 days for pointings searched to a DM of $500 \mathrm{pc} \mathrm{cm}^{-3}$. The latter pointings are sensitive only to FRBs with low extragalactic DM contributions. Thus, we are unevenly sampling the range of extragalactic DMs for the pointings we have searched, implying an uneven coverage of potential FRBs. However, if we assume that all values of extragalactic DM contribution are equally likely, we can estimate an upper limit using the total observing time of 84 days that includes both pointings searched to a DM of 3000 and $500 \mathrm{pc} \mathrm{cm}^{-3}$.

For the flux limit $S_{\min }=0.63 \mathrm{Jy}$ corresponding to the fieldof-view-averaged gain of $1.44 \mathrm{~K} / \mathrm{Jy}$ for the FWHM case, we estimate a $95 \%$ confidence upper limit on the FRB rate of

$$
\begin{aligned}
& R<4.98 \times 10^{3} \mathrm{FRBs} \mathrm{sky}^{-1} \mathrm{day}^{-1}(T=61 \text { days }) \\
& R<3.62 \times 10^{3} \mathrm{FRBs} \mathrm{sky}^{-1} \mathrm{day}^{-1}(T=84 \text { days })
\end{aligned}
$$

and for the flux limit $S_{\min }=0.76$ Jy corresponding to the fieldof-view-averaged gain of $1.19 \mathrm{~K} / \mathrm{Jy}$ for the Parkes-equivalent case, we obtain

$$
\begin{aligned}
& R<3.03 \times 10^{3} \mathrm{FRBs}^{-1} \mathrm{day}^{-1}(T=61 \text { days }) \\
& R<2.20 \times 10^{3} \mathrm{FRBs}^{-1} \mathrm{day}^{-1}(T=84 \text { days })
\end{aligned}
$$

The survey is ongoing, with $\sim 50,000$ pointings left to be observed in order to cover the GBT visible sky. A nondetection in these pointings will improve the constraint on the rate to $1.98 \times 10^{3} \mathrm{FRBs} \mathrm{sky}^{-1} \mathrm{day}^{-1}$ for the FWHM case.

\subsection{Estimation of Volumetric Rate}

We can also constrain the volumetric rate of FRBs up to the redshift out to which the GBNCC survey searches. For each pointing, we are searching out to a different redshift as the DM contribution from the Galaxy varies greatly across the sky. We estimate the DM due to our Galaxy, $\mathrm{DM}_{\mathrm{MW}}$, as the maximum line-of-sight DM predicted by the NE2001 model for each of our pointings. The DM contribution of the IGM can be estimated using the following equation:

$$
\mathrm{DM}_{\mathrm{IGM}}=\mathrm{DM}_{\text {thresh }}-\left(\frac{\mathrm{DM}_{\text {host }}}{z+1}+\mathrm{DM}_{\mathrm{MW}}\right) \text {. }
$$

Here $\mathrm{DM}_{\text {thresh }}$ is the maximum DM searched by the analysis pipeline, either $3000 \mathrm{pc} \mathrm{cm}^{-3}$ or $500 \mathrm{pc} \mathrm{cm}^{-3}$. Assuming the electron density distribution of the potential host galaxy of the FRB progenitor to be similar to that of our Galaxy, we obtain a

\footnotetext{
${ }^{19}$ https://science.nrao.edu/facilities/gbt/proposing/GBTpg.pdf
} 
DM contribution for the host galaxy, $\mathrm{DM}_{\text {host }}=80 \mathrm{pc} \mathrm{cm}^{-3}$, by averaging over the maximum DM predicted by the NE2001 model for evenly spaced lines of sight through our Galaxy. However, we assume $\mathrm{DM}_{\text {host }}$ as $100 \mathrm{pc} \mathrm{cm}^{-3}$ for evaluating the limiting redshift of the GBNCC survey, following Thornton et al. (2013). The above assumption is to allow for a meaningful comparison with the redshifts of 0.5 to 1 inferred by Thornton et al. (2013) for four FRBs discovered with the Parkes telescope. The assumption for $\mathrm{DM}_{\text {host }}$ is reduced by a factor of $(z+1)$ to facilitate comparison with the effect of $\mathrm{DM}_{\mathrm{MW}}$ and $\mathrm{DM}_{\mathrm{IGM}}$ (Ioka 2003). The reduction in the DM of the host galaxy accounts for the decrease in the observed frequency by a factor of $(z+1)$ as compared to the emission frequency of a source at a redshift $z$ and the increase in the observed dispersive delay by a factor of $(z+1)$. The limiting redshift, $z$, for each pointing can be determined using the DMredshift relation, $\mathrm{DM}_{\mathrm{IGM}}=1200 z \mathrm{pc} \mathrm{cm}^{3} \quad$ (Ioka 2003; Inoue 2004). We find the mean limiting redshift, $z_{\lim }=1.84$, for the GBNCC pointings included in our FRB search.

We note that there are significant uncertainties in the DMredshift relation used for the estimation of the limiting redshift. However, the relation is corroborated by the determination of the redshift of the repeating FRB121102 and the resulting estimate of the DM of its host galaxy. The DM obtained for the host galaxy, after subtracting the Galactic DM and the DM contribution estimated for the IGM using the DM-redshift relation, is equivalent to that expected from a dwarf galaxy (Tendulkar et al. 2017). The observations of Tendulkar et al. (2017) also imply that the assumption of $\mathrm{DM}_{\text {host }}=100 \mathrm{pc} \mathrm{cm}^{-3}$ could be an underestimate if FRBs preferentially exist in dwarf galaxies. The mean limiting redshift for the GBNCC pointings reduces to $z_{\text {lim }}=1.79$, if we assume $D_{\text {host }}$ to be equal to the upper limit on the inferred DM of the host galaxy of FRB121102 $\left(225 \mathrm{pc} \mathrm{cm}^{-3}\right)$. The estimate of the mean limiting redshift is thus not sensitive to the assumption for the DM contribution of the host galaxy.

We then estimate the comoving volume surveyed by each of the pointings using the solid angle for the GBT beam at $350 \mathrm{MHz}, \Omega=0.408 \mathrm{deg}^{2}$, and assuming Planck 2015 cosmological parameters (Ade et al. 2016). The total comoving volume searched by the survey is estimated by summing up the comoving volume for all the pointings and is equal to $3.8 \times 10^{11} \mathrm{Mpc}^{3}$. We note that the above estimate is an upper limit at best since the comoving volume surveyed at $350 \mathrm{MHz}$ is flux limited and cannot be correctly determined by the maximum DM searched. The intrinsic luminosity distribution of FRBs could be such that FRBs at high redshifts have flux densities less than the survey sensitivity. Additionally, pulses from high-redshift FRBs, whose intrinsic luminosity does not limit detectability, can be broadened by intrachannel and DM step smearing. Since the threshold flux density determined by Equation (1) depends on the broadened pulse width, which increases with increasing redshift, high-redshift FRBs with correspondingly higher DMs are harder to detect, which can also cause our survey in this volume to be flux limited.

The upper limit on the FRB rate per unit comoving volume inferred using our upper limit on the sky rate for the FWHM case of $3.6 \times 10^{3} \mathrm{FRBs} \mathrm{sky}^{-1} \mathrm{day}^{-1}$ is $3.5 \times 10^{3} \mathrm{Gpc}^{-3} \mathrm{yr}^{-1}$, for isotropic emission. The rate reported here is valid up to the mean limiting redshift for the GBNCC pointings, $z_{\text {lim }}=1.84$, and under the assumptions that the population of FRBs does

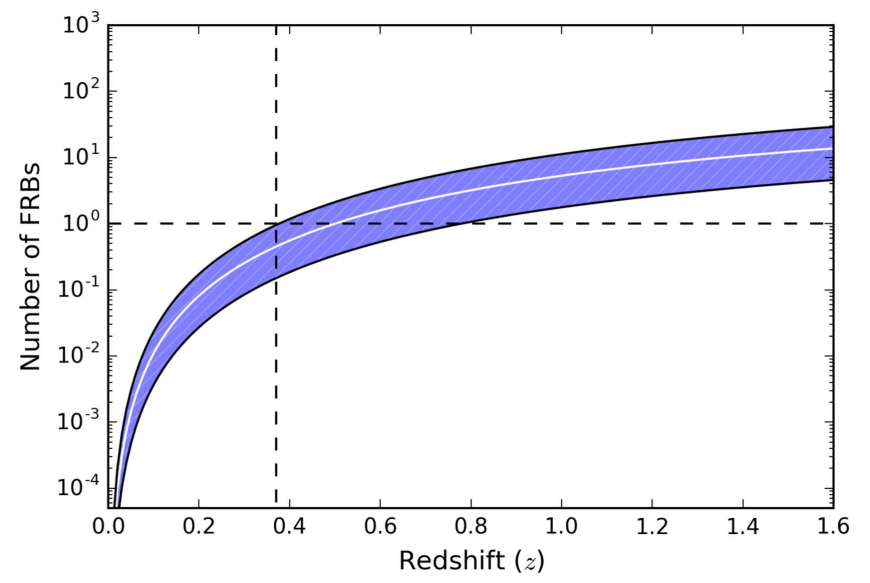

Figure 5. Number of FRBs expected to be detected with the observing time and sky coverage of the GBNCC survey for different limiting redshifts. The white curve is obtained by scaling the rate reported by Crawford et al. (2016) with comoving volume, and the hatched region represents $99 \%$ bounds on the rate. The GBNCC survey searched out to a limiting redshift of 0.37 , as the rate for the Parkes surveys predicts detection of 1 FRB with GBNCC at $z=0.37$.

not evolve with redshift and that all FRBs located at $z<z_{\text {lim }}$ are detectable with GBNCC. The rate could be an underestimate if FRBs exhibit beamed radio emission. This is possible if FRBs are extragalactic, as the extremely high implied brightness temperatures in that scenario would suggest that the emission is coherent and beamed.

The survey's limiting redshift and the corresponding upper limit on the volumetric rate can also be estimated following the method and assumptions detailed in Rajwade \& Lorimer (2017). The rate estimate of $3.3 \times 10^{3} \mathrm{FRBs} \mathrm{sky}^{-1} \mathrm{day}^{-1}$ above a fluence of $3.8 \mathrm{Jy} \mathrm{ms}$ for the Parkes surveys reported by Crawford et al. (2016) is assumed to be survey independent and valid at a frequency of $350 \mathrm{MHz}$ and for a limiting redshift of $z_{\lim }=0.75$ (Lorimer et al. 2013). The limiting redshift of 0.75 is an assumption based on the redshifts of $0.5-1$ inferred from the DMs of the FRBs discovered by Thornton et al. (2013). We translate the Parkes rate to a range of redshifts by assuming a constant comoving number density distribution of FRBs. We compute the number of FRBs detectable by GBNCC for a range of limiting redshifts using the corresponding Parkes rate. The number detectable with GBNCC for an observing time of 84 days is represented by the white curve shown in Figure 5. The limiting redshift, $z_{\lim }=0.37$, is the one for which the GBNCC survey is predicted to detect 1 FRB. The conclusion is justified because if the survey were sensitive to a redshift greater than $z_{\text {lim }}$, then the Parkes rate estimate predicts a detection with the GBNCC survey, which is inconsistent with our observations.

The above two limiting redshift estimates, obtained using different approaches, depend on several different assumptions that cannot currently be tested. The large discrepancy between the two redshift estimates can be explained if the comoving volume estimated for the first case (hereafter case A) is flux limited such that FRBs located at $z<1.84$ are not detectable even though we are searching the DM range extending out to $z_{\lim }=1.84$. The estimate of the limiting redshift for the second case (hereafter case B) is thus more robust since it is based on the GBNCC survey sensitivity and the underlying assumption of FRBs being standard candles, which ensures that all sources in the estimated comoving volume are detectable. 
Assuming the redshift estimate of 0.37 to be correct, we conclude that the upper limit on the volumetric rate is $1.6 \times 10^{5} \mathrm{Gpc}^{-3} \mathrm{yr}^{-1}$, with the caveat that treating the $1.4 \mathrm{GHz}$ rate estimate as an all-sky rate at $350 \mathrm{MHz}$ involves the implied assumption of a flat spectral index. Obtaining the rate at $350 \mathrm{MHz}$ by scaling with a different assumed spectral index would change the estimate of the limiting redshift and volumetric rate. The estimate is also sensitive to the assumed intrinsic luminosity distribution and would vary if, instead of the standard candle assumption, a distribution of luminosities were assumed.

\section{Spectral Index Constraints}

Observations of FRBs can help determine the intrinsic spectral index if the position of the FRB within the telescope beam is known. Keane et al. (2016) measured $\alpha=1.3 \pm 0.5$ for FRB150418 assuming that the FRB is located at the position of the potentially associated variable source found within the Parkes beam. The association has, however, been questioned by Williams \& Berger (2016) and Vedantham et al. (2016b). The intrinsic spectral index can also be constrained by methods other than observation and localization. In this section, we use the nondetection with GBNCC to constrain the spectral index for different astrophysical scenarios.

We perform Monte Carlo simulations for FRB flux distributions consistent with the rate estimate reported at $1.4 \mathrm{GHz}$ for the Parkes surveys, $3.3 \times 10^{3} \mathrm{FRBs} \mathrm{sky}^{-1} \mathrm{day}^{-1}$ (Crawford et al. 2016). We assume a power-law flux density model for FRBs with flux density at a frequency $\nu, S_{\nu} \propto \nu^{\alpha}$. The cumulative flux density distribution function (the $\log N-$ $\log S$ function) of the FRB population is also modeled as a power law with an index $\gamma$. This implies that the number of FRBs with a flux density greater than $S$ is

$$
N(>S) \propto S^{-\gamma} \text {. }
$$

For a nonevolving population uniformly distributed in a Euclidean universe, $\gamma=1.5$, for any luminosity distribution. Any value other than 1.5 would argue for FRBs being a cosmological population and/or exhibiting redshift-dependent evolution. Vedantham et al. (2016a) calculate $\gamma$ based on multiple-beam detections with Parkes and different detection rates for varying dish diameters, and they report a constraint, $0.66<\gamma<0.96$. Oppermann et al. (2016) derive the constraint $0.8 \leqslant \gamma \leqslant 1.7$ making use of the detections with the HTRU survey at Parkes and the PALFA Survey at Arecibo. We use three different values of the slope of the $\log N-\log S$ function $(\gamma=0.8,1.2$, and 1.5) for our simulations to roughly sample the range in which it is estimated to vary.

\subsection{Absence of Scattering and Free-Free Absorption}

To reconcile the upper limit on the FRB rate obtained from GBNCC with the observed rate from the Parkes surveys, it may be that FRBs are rendered undetectable at low frequencies by scattering and/or the presence of a spectral turnover, either intrinsic to the emission mechanism or due to free-free absorption. In the absence of scattering and free-free absorption, the intrinsic spectral index needs to be relatively flat or even positive to account for our nondetection.

We ran 100 Monte Carlo iterations each for different cumulative flux density distributions $(\gamma=0.8,1.2$, and 1.5). For each Monte Carlo iteration, we generated a flux density distribution of FRBs at $1.4 \mathrm{GHz}$ consistent with the rate for

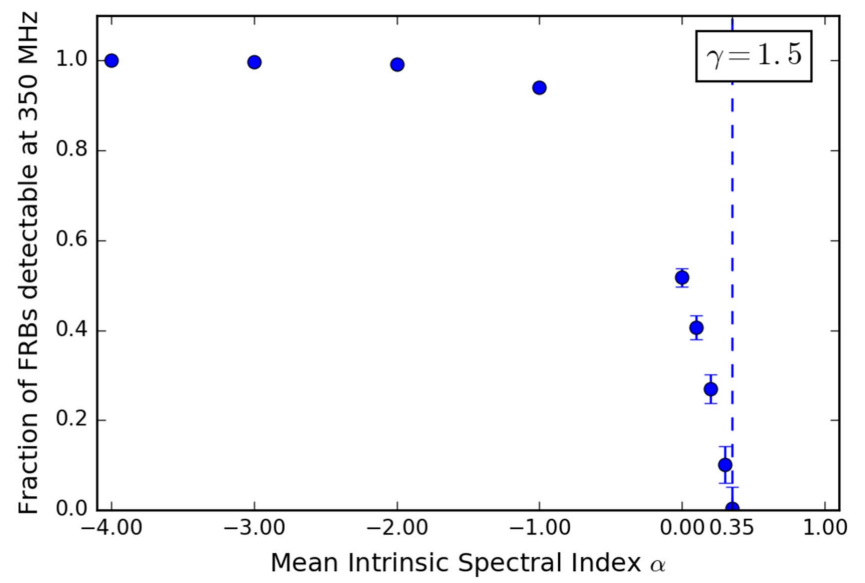

Figure 6. Number of GBNCC-detectable FRBs plotted as a fraction of the computed all-sky FRB rate for GBNCC assuming an FRB population consistent with the $1.4 \mathrm{GHz}$ Parkes rate estimate. Any spectral index less than the constraint, $\alpha_{\text {lim }}$, can be rejected, as it predicts FRB detections with GBNCC. The dashed line marks the constraint, $\alpha_{\lim }=0.35$, for a Euclidean flux distribution $(\gamma=1.5)$. The error bars correspond to $3 \sigma$ uncertainties, and the plotted spectral indices correspond to the mean of a normal distribution with a width of 0.5 .

Table 2

Spectral Index Constraints

\begin{tabular}{lccc}
\hline \hline$\gamma$ & \multirow{2}{*}{ No Scattering/FF } & \multicolumn{2}{c}{ Scattering $^{\mathrm{b}}$} \\
\cline { 3 - 4 } & & Crawford et al. & Champion et al. \\
\hline 0.8 & $>0.19$ & $>-0.9$ & $>-1.5$ \\
1.2 & $>0.28$ & $>-0.6$ & $>-1.2$ \\
1.5 & $>0.35$ & & $>-0.9$ \\
\hline
\end{tabular}

Notes.

${ }^{\mathrm{a}} \mathrm{FF}$ refers to free-free absorption.

$\mathrm{b}$ The two columns correspond to different $1.4 \mathrm{GHz}$ rate estimates assumed for the initial flux distribution.

Parkes surveys. We scaled the distribution to $350 \mathrm{MHz}$ by sampling the spectral index of each FRB from a normal distribution $(\sigma=0.5)$ centered on the mean spectral index $\alpha$ ranging from -4 to +1 . From the resulting flux distribution, we computed the all-sky rate of FRBs above a peak flux density of $0.63 \mathrm{Jy}$ at $350 \mathrm{MHz}$. Figure 6 shows the number of GBNCCdetectable FRBs, i.e., the difference of the computed all-sky FRB rate and the $95 \%$ confidence GBNCC upper limit as a fraction of the computed all-sky FRB rate, for a range of spectral indices.

The constraining spectral index is the one for which the computed all-sky rate was found to be equal to the $95 \%$ confidence GBNCC upper limit, i.e., when simulations do not predict any detections in the absence of scattering and free-free absorption. The constraints on the mean spectral index for different values of $\gamma$ are listed in Table 2. The strongest constraint, $\alpha>0.35$, was obtained for a Euclidean flux distribution $(\gamma=1.5)$. The constraint depends on the assumed width of the distribution of spectral indices since the detectable FRBs in each distribution will be those with lower spectral indices. Therefore, decreasing the width will weaken the constraint on the mean spectral index. In the event of all FRBs having the same spectral index, we derive the constraint $\alpha>0.09$, for $\gamma=1.5$. 


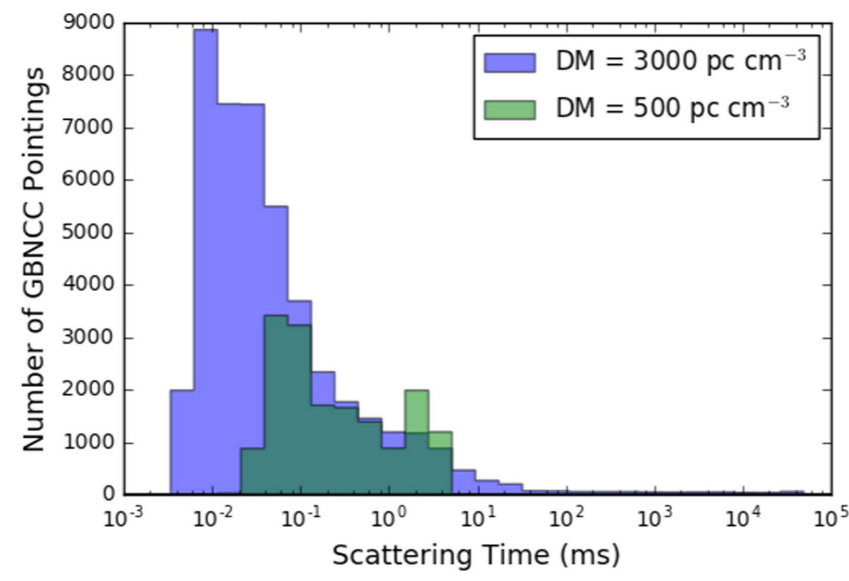

Figure 7. Scattering timescales at $350 \mathrm{MHz}$ predicted by the NE2001 model for all GBNCC pointings included in the FRB search. The maximum possible scattering timescales for our Galaxy along the line of sight of the GBNCC pointings are plotted here, thus assuming that the burst traverses the entire Galactic column modeled by the NE2001 model along that line of sight. The pointings in blue have been searched to a DM of $3000 \mathrm{pc} \mathrm{cm}^{-3}$, and the pointings in green have been searched to a DM of $500 \mathrm{pc} \mathrm{cm}^{-3}$.

\subsection{Scattering}

Scattering may arise from three sources: our Galaxy, the IGM, and the host galaxy. Figure 7 shows the scattering times at $350 \mathrm{MHz}$ predicted by the NE2001 model along the lines of sight of all GBNCC pointings that were searched for FRBs. Since the scattering time for $98 \%$ of these pointings is less than $10 \mathrm{~ms}$ (much less than our maximum searched boxcar width; see above), we can assume that the scattering from Galactic structures, which are modeled by the NE2001 model, is not responsible for smearing all potentially GBNCC-detectable FRBs beyond detection. However, compact regions of high electron density in our Galaxy, which are not accounted for by the NE2001 model, can potentially result in scattering timescales greater than $10 \mathrm{~ms}$.

Masui et al. (2015) argue against the IGM being the dominant source of scattering and support the hypothesis of strong scattering from either the dense central region of the host galaxy or a compact nebula surrounding the source. This conclusion is derived from FRB110523 showing evidence of being scattered by two plasma screens and exhibiting strong scintillation. Katz (2016) found no correlation between the measured pulse widths of FRBs and their extragalactic DMs, suggesting that the IGM does not contribute to both scattering and extragalactic DM.

Having established the contribution to scattering from the IGM and the Galactic structures modeled by the NE2001 model as being irrelevant for our nondetection, we ran our simulations with a three-parameter lognormal distribution of scattering times. The parameters of this distribution were chosen on the basis of the distribution of Earth-centered scattering times for our Galaxy to allow for both source models supported by Masui et al. (2015), namely, a dense nebula local to the source or location in the central region of the host galaxy. The threshold parameter defines the minimum of the distribution and is set to be equal to the minimum Earth-centered scattering time for our Galaxy, $4.3 \times 10^{-3} \mathrm{~ms}$. The scale of the distribution was set as a free parameter to allow for a range of values of the mean. The standard deviation, $\sigma=2.74 \mathrm{~ms}$, of the underlying normal distribution was set to be the same as

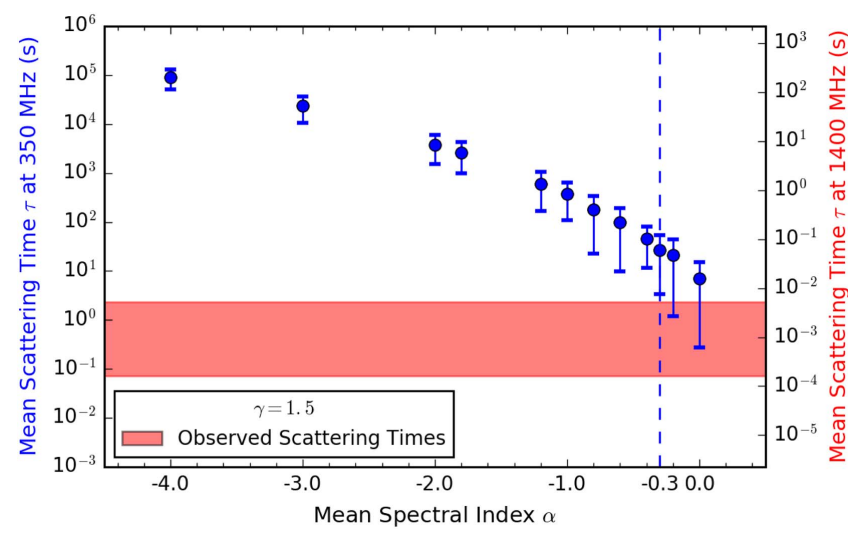

Figure 8. Mean scattering time of the lognormal distribution that would render FRBs with a particular spectral index undetectable with GBNCC. The dashed line marks the spectral index constraint, $\alpha_{\text {lim }}=-0.3$, for a Euclidean flux distribution $(\gamma=1.5)$. The $x$-axis represents the mean of a normal distribution of spectral indices with a width of 0.5 . The pink shaded band corresponds to the observed range of scattering times (Cordes et al. 2016) at $1.4 \mathrm{GHz} .2 \sigma$ error bars have been plotted.

that of the distribution of scattering times for our Galaxy at $350 \mathrm{MHz}$ predicted by the NE2001 model.

As in Section 5.1, we generated a flux density distribution at 1.4 $\mathrm{GHz}$ and scaled it to $350 \mathrm{MHz}$ using spectral indices drawn from a normal distribution centered on the mean spectral index $(-4<\alpha<0)$. We estimated the threshold flux density of the GBNCC survey to be $0.82 \mathrm{Jy}$ for $t_{\text {scatt }}=10 \mathrm{~ms}$, accounting for the contribution to scattering from the IGM and our Galaxy. FRBs in the flux distribution that are detectable with GBNCC $(S>0.82 \mathrm{Jy})$ were assigned scattering times drawn from the above-mentioned lognormal distribution. This step was repeated with the mean of the lognormal distribution increased for each repetition until the scattering timescales of all detectable FRBs became greater than $100 \mathrm{~ms}$. Since the widest boxcar template used by our search pipeline for detecting single pulses is $100 \mathrm{~ms}$ (see Section 3), FRBs with a scattering timescale greater than $100 \mathrm{~ms}$ will not be detected with an optimal $\mathrm{S} / \mathrm{N}$ by our search pipeline. The above analysis assumes uniform sensitivity to pulses of any scattering timescale less than $100 \mathrm{~ms}$. Although there is a reduced sensitivity to highly scattered pulses because of the prevalence of RFI on longer timescales, the effect is countered by the reduction in the minimum peak $\mathrm{S} / \mathrm{N}$ required to satisfy RRATtrap's cluster requirement with increase in the scattering timescale, as shown in Figure 4.

Figure 8 shows the mean scattering time of the lognormal distribution that can render FRBs in the flux density distribution expected to be seen by GBNCC (with $S>0.82 \mathrm{Jy}$ ) in an observing time of 84 days undetectable, for a range of spectral indices. A more negative spectral index would predict a higher number of detections with GBNCC, requiring a higher mean scattering time at $350 \mathrm{MHz}$ to render all the FRBs undetectable. We find our constraint on the spectral index, $\alpha_{\text {lim }}$, to be the one for which the mean scattering time at $350 \mathrm{MHz}$ scales to the maximum observed scattering timescale for known FRBs at $1.4 \mathrm{GHz}$ assuming a Kolmogorov scaling. We derive the constraint, $\alpha_{\lim }>-0.3$, for a Euclidean flux distribution. This constraint is valid only in the absence of free-free absorption. The constraints for other values of $\gamma$ are listed in Table 2 . 
Different surveys conducted at $1.4 \mathrm{GHz}$ with the Parkes telescope have different reported rate estimates and flux density thresholds. To gauge the sensitivity of our results to the assumed $1.4 \mathrm{GHz}$ rate estimate, we repeat this analysis with a flux distribution at $1.4 \mathrm{GHz}$ that is consistent with the rate reported by Champion et al. (2016) of $7 \times 10^{3}$ FRBs sky $^{-1}$ day $^{-1}$ above a flux density of $0.17 \mathrm{Jy}$ for $W_{\mathrm{i}}=5 \mathrm{~ms}$. The resulting constraints are weaker and are listed in Table 2. The constraints on spectral index are also sensitive to the width of the lognormal distribution. Decreasing the width of the distribution would allow even modest scattering times to explain our nondetection, thus weakening the constraints on spectral index.

Another effect that can potentially weaken our constraints is the $1.4 \mathrm{GHz}$ observation of a reduced FRB detection rate at low and intermediate Galactic latitudes as compared to high Galactic latitudes by Petroff et al. (2014). A recent analysis by Vander Wiel et al. (2016) demonstrates that the reduction in the FRB rate is significant $\left(p=5 \times 10^{-5}\right)$ for low Galactic latitudes $\left(|\mathrm{b}|<5^{\circ}\right)$, while the difference between the midlatitude $\left(5^{\circ}<|\mathrm{b}|<15^{\circ}\right)$ and the high-latitude FRB rate is only marginally significant $(p=0.03)$. Since only $5 \%$ of the GBNCC survey pointings were observed at Galactic latitudes $|\mathrm{b}|<5^{\circ}$, and $10 \%$ of the pointings were observed at intermediate Galactic latitudes, incorporating the latitude dependence of the FRB rate would not have a significant effect on the resulting constraints. Additionally, if the latitude dependence of the FRB event rate is due to diffractive scintillation, as suggested by Macquart \& Johnston (2015), then the frequency dependence of this effect can also weaken the spectral index constraints. However, we do not account for this effect here since Scholz et al. (2016) demonstrate that the analysis by Macquart \& Johnston (2015) is incorrect, as its prediction for a high FRB rate with the PALFA survey is not matched by observations.

\subsection{Constant Comoving Number Density Distribution}

We attempt to constrain the spectral index for the specific case of a constant comoving number density distribution in this section. The approach follows from the analysis in Rajwade \& Lorimer (2017, and references therein) and enables us to derive constraints for a variety of astrophysical models. It is based on the assumption that FRBs are standard candles, thus making it different from the approach described in Section 5.2.

The bolometric luminosity, $L$, for each model and spectral index $\alpha$ is evaluated using the following equation assuming an $S_{\text {peak }}=1$ Jy detection of an FRB located at $z_{\text {lim }}=0.75$ (Lorimer et al. 2013) with the Parkes surveys:

$$
S_{\text {peak }}=\frac{L \int_{\nu_{1}^{\prime}}^{\nu_{2}^{\prime}} E_{\nu^{\prime}} d \nu^{\prime}}{(1+z)^{2} 4 \pi D(z)^{2}\left(\nu_{2}-\nu_{1}\right) \int_{\nu_{\text {low }}^{\prime}}^{\nu_{\text {high }}^{\prime}} E_{\nu^{\prime}}^{\prime} d \nu^{\prime}}
$$

Here $D(z)$ is the comoving distance calculated using Planck 2015 cosmological parameters (Ade et al. 2016), and $\nu^{\prime}=(1+z) \nu$ is the frequency in the source frame. The limiting frequencies for emission, $\nu_{\text {high }}^{\prime}$ and $\nu_{\text {low }}^{\prime}$, are assumed to be $10 \mathrm{GHz}$ and $10 \mathrm{MHz}$, respectively (Lorimer et al. 2013). The frequencies $\nu_{1}$ and $\nu_{2}$ are the lowest and highest observing frequencies, respectively, of the survey in consideration.
Table 3

Spectral Index Constraints for Constant Comoving Number Density Distribution

\begin{tabular}{|c|c|c|c|}
\hline Model & $\begin{array}{c}T_{\mathrm{e}} \\
(\mathrm{K})\end{array}$ & $\begin{array}{c}\mathrm{EM} \\
\left(\mathrm{cm}^{-6} \mathrm{pc}\right)\end{array}$ & $\alpha_{\lim }$ \\
\hline A & $\ldots$ & $\ldots$ & 1.18 \\
\hline B & $\ldots$ & $\ldots$ & -2.48 \\
\hline $\mathrm{C}$ & 200 & 1000 & 1.00 \\
\hline D & 8000 & $1.5 \times 10^{6}$ & -0.64 \\
\hline E & 200 & 1000 & -2.67 \\
\hline $\mathrm{F}$ & 8000 & $1.5 \times 10^{6}$ & -4.39 \\
\hline
\end{tabular}

The bolometric luminosity is different for each model because of the difference in the expression for the energy released per unit frequency interval, $E_{\nu^{\prime}}$. In the absence of scattering and free-free absorption, positive spectral indices will be the sole reason for reduction of flux at low frequencies, and we can set $E_{\nu^{\prime}} \propto \nu^{\prime \alpha}$. Mirroring the terminology used by Rajwade \& Lorimer (2017), we will be referring to it as model A hereafter. For the model where scattering becomes important (model B), $E_{\nu^{\prime}}$ gets reduced by a factor of $\sqrt{1+\left(t_{\text {scatt }} / W_{\mathrm{i}}\right)^{2}}$. Here $t_{\text {scatt }}$ is the scattering timescale at a frequency $\nu$ obtained by scaling the mean observed timescale of $6.7 \mathrm{~ms}$ at $1 \mathrm{GHz}$ under the assumption of a Kolmogorov scattering spectrum. The observed scattering time of $6.7 \mathrm{~ms}$ was determined by taking the average of the scattering timescales of known FRBs (Cordes et al. 2016). For FRBs with no measured scattering timescales, we used half of the published upper limits when computing the average.

Another astrophysical phenomenon that can render FRBs undetectable at low frequencies is free-free absorption in the dense environment surrounding the FRB progenitor. For the case of free-free absorption,

$$
E_{\nu^{\prime}} \propto\left(\frac{\nu^{\prime}}{1 \mathrm{GHz}}\right)^{\alpha} \exp \left[-\tau\left(\frac{\nu^{\prime}}{1 \mathrm{GHz}}\right)^{-2.1}\right] .
$$

The optical depth, $\tau$, at $1 \mathrm{GHz}$ is computed using $\tau=0.082$ $T_{\mathrm{e}}^{-1.35}$ EM (Mezger \& Henderson 1967), where $T_{\mathrm{e}}$ is the electron temperature and EM is the emission measure. We considered two models of free-free absorption: cold molecular clouds with ionization fronts (model $\mathrm{C}$ ), and hot, ionized magnetar ejecta/circumburst medium (model D). The parameters $T_{\mathrm{e}}$ and EM for these models have been adopted from Rajwade \& Lorimer (2017) and are listed in Table 3. Models $\mathrm{E}$ and $\mathrm{F}$ mimic models $\mathrm{C}$ and $\mathrm{D}$, respectively, but also account for scattering. For this, the expression for $E_{\nu^{\prime}}$ in Equation (10) is reduced by a factor of $\sqrt{1+\left(t_{\text {scatt }} / W_{\mathrm{i}}\right)^{2}}$, as was done for model B.

Using the expressions for $E_{\nu^{\prime}}$ derived above, we calculate the peak flux density detectable with the GBNCC survey for each model and spectral index $\alpha$ by substituting the bolometric luminosity for that model and spectral index and parameters of the GBNCC survey in Equation (9). This calculation is performed for a range of redshifts. The peak flux density at the GBNCC survey's limiting redshift for case $\mathrm{B}, z_{\mathrm{lim}}=0.37$, should be equal to the survey's sensitivity for the constraining spectral index $\alpha_{\text {lim. }}$. Any spectral index $\alpha<\alpha_{\text {lim }}$ can be rejected, as the peak flux density at $z_{\lim }$ for $\alpha<\alpha_{\lim }$ would be greater than the survey sensitivity, implying FRB detections 


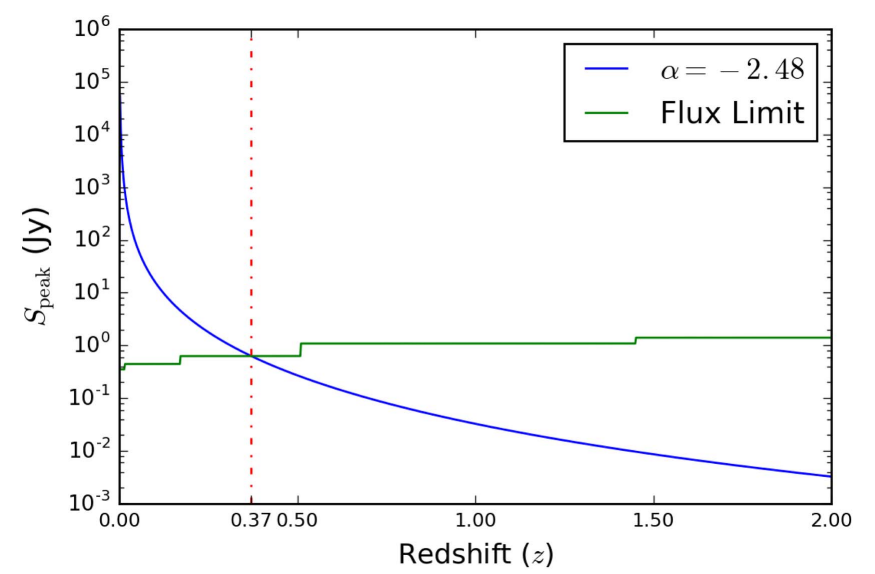

Figure 9. Peak flux density as a function of redshift for the constraining spectral index, $\alpha_{\text {lim }}$, for model B (scattering). The green line corresponds to the minimum detectable flux density for the GBNCC survey, and the red line marks the limiting redshift for the GBNCC survey, $z_{\text {lim }}=0.37$, for case $B$. The peak flux density for the constraining spectral index, evaluated using Equation (9) and represented by the blue curve, is shown to be equal to the survey sensitivity at the limiting redshift.

with the GBNCC survey. The procedure is shown graphically in Figure 9, and the resulting constraints are listed in Table 3.

The GBNCC survey sensitivity exhibits a nonlinear dependence on redshift. If the IGM is assumed to be the dominant contributor to the DM, then the DM-redshift relation (Ioka 2003; Inoue 2004) implies that we are searching for FRBs with higher DMs as we search out to higher redshifts. The increase in DM increases the dispersive smearing within each frequency channel (evaluated using Equation (3)), thereby broadening the pulse and increasing the minimum detectable flux density of the survey. The survey sensitivity for the broadened pulse width is determined using Equation (1) and is plotted in Figure 9.

The constraints listed in Table 3 are based on the limiting redshift for the GBNCC survey for case B, the calculation of which is based on the assumption that the Parkes surveys searched to a redshift $z_{\text {lim }}=0.75$. If the repeating FRB121102 is not representative of the FRB population, i.e., not all FRBs are cosmological, then this assumption might not hold true. Other caveats associated with these constraints have been detailed in Rajwade \& Lorimer (2017).

For the case of scattering, the constraint for a uniform distribution in comoving volume of FRBs, $\alpha_{\text {lim }}=-2.48$, is very weak in comparison with $\alpha_{\text {lim }}=-0.3$ evaluated for a Euclidean flux distribution with the approach described in Section 5.2. The constraint for a Euclidean flux distribution is derived by assuming a distribution of scattering times as compared to a single scattering time for all FRBs assumed for evaluating the weaker constraint. The marked difference in the resulting constraints points to the sensitivity of our results to the initial assumptions about the scattering timescale.

The constraints based on the GBNCC nondetection are not markedly different from the constraints evaluated by Rajwade \& Lorimer (2017) based on nondetection with surveys such as AO327 (Deneva et al. 2016), LOFAR (Karastergiou et al. 2015), and UTMOST (Caleb et al. 2016). The constraint we derive under the assumption of absence of scattering and freefree absorption, $\alpha_{\lim }=1.18$, is stronger than the most constraining spectral index obtained from the above-mentioned surveys $\left(\alpha_{\text {lim }}=0.7 ; \mathrm{AO} 327\right)$. The best constraint derived by
Rajwade \& Lorimer (2017) for the model where scattering becomes relevant, $\alpha_{\text {lim }}=-2.10$, is based on nondetection with UTMOST and is stronger than the constraint obtained using the GBNCC nondetection, $\alpha_{\text {lim }}=-2.48$.

\section{Implications for Other Surveys}

We can predict the FRB detection rates for current and upcoming surveys using the constraints on spectral index derived from GBNCC. We derive the following equation for the calculation of the FRB rate $R$ above a flux density $S_{0}$ at a frequency $\nu_{0}$, for a spectral index $\alpha$ and slope of the $\log N-\log$ $S$ function $\gamma$ :

$$
\begin{aligned}
R\left(>S_{0}\right) & =R_{\text {ref }}\left(\frac{S_{0}}{S_{\text {ref }}\left(\frac{\nu_{0}}{\nu_{\text {ref }}}\right)^{\alpha}}\right)^{-\gamma} \\
& =R_{\text {ref }}\left(\frac{S_{0}}{S_{\text {ref }}}\right)^{-\gamma}\left(\frac{\nu_{0}}{\nu_{\text {ref }}}\right)^{\alpha \gamma} .
\end{aligned}
$$

Here $R_{\text {ref }}$ is the reference rate estimate above a flux density $S_{\text {ref }}$ at a frequency $\nu_{\text {ref }}$. The above equation uses a scaling factor of $\nu^{\alpha \gamma}$ to calculate the FRB rate instead of the $\nu^{\alpha}$ used by BurkeSpolaor \& Bannister (2014). The correction to the scaling factor can be justified in the following manner. If $R_{\text {ref }}$ is the number of bursts detectable per sky per day above a flux density $S_{\text {ref }}$ at a frequency $\nu_{\text {ref }}$, then $R_{\text {ref }}$ is also the number of bursts detectable above a flux density $S_{\text {ref }}\left(\nu_{0} / \nu_{\text {ref }}\right)^{\alpha}$ at a frequency $\nu_{0}$. The ratio of the number of bursts $R$ detectable above a flux density $S_{0}$ and the number of bursts $R_{\text {ref }}$ detectable above a flux density $S_{\text {ref }}\left(\frac{\nu_{0}}{\nu_{\text {ref }}}\right)^{\alpha}$ can then be given by Equation (11).

However, Equation (11) makes incorrect assumptions about the FRB population in that it does not allow a distribution of spectral indices and scattering timescales. This warrants the need to run Monte Carlo simulations to ensure that the predicted rate accounts for the occasional bright FRBs with scattering times lower than the mean of the population. For instance, all FRBs with a scattering time greater than $1 \mathrm{~ms}$ at $1 \mathrm{GHz}$ will not be detectable with an optimal $\mathrm{S} / \mathrm{N}$ with the GBNCC survey. This is because the widest boxcar template of $100 \mathrm{~ms}$ used by our search pipeline corresponds to a timescale of $1 \mathrm{~ms}$ at $1 \mathrm{GHz}$ (under the assumption of a Kolmogorov medium). However, scattering timescales of known FRBs at $1 \mathrm{GHz}$ range from 0.7 to $23 \mathrm{~ms}$, with several of these measurements being upper limits (Cordes et al. 2016), suggesting that the survey could still be sensitive to a significant fraction of the FRB population.

We generated flux distribution of FRBs at $1.4 \mathrm{GHz}$ consistent with the Parkes rate estimate for $\gamma=0.8,1.2$, and 1.5. Spectral indices drawn from a normal distribution $(\sigma=0.5)$ centered on the mean spectral index were used to scale the flux distribution to the frequency of the survey in consideration. For each FRB in the distribution, the scattering time $t_{350}$ was sampled from a lognormal distribution at $350 \mathrm{MHz}$, with the width the same as our Galaxy's distribution. The mean of the lognormal distribution was set to be the scattering timescale at $350 \mathrm{MHz}$ obtained by scaling the mean observed timescale of $6.7 \mathrm{~ms}$ at $1 \mathrm{GHz}$ assuming a Kolmogorov spectrum. The flux of all FRBs in the distribution was reduced by $\sqrt{1+\left(t_{\text {scatt }} / W_{\mathrm{i}}\right)^{2}}$, where $t_{\text {scatt }}$ was the scattering time for 
each FRB at the survey frequency, obtained by scaling $t_{350}$, again under the assumption of a Kolmogorov spectrum. The number of FRBs in this distribution with a flux greater than $S_{0}$ was used to compute the number of bursts per hour detectable by the survey. The minimum detectable flux density for each survey, $S_{0}$, was evaluated using Equation (1) with $(\mathrm{S} / \mathrm{N})_{\mathrm{b}}=10, t_{\text {scatt }}=0$ and the parameters for the surveys considered listed in Table 1.

Our simulations predict the rate for mean spectral indices ranging from $\alpha_{\text {lim }}$ to an arbitrary upper limit, $\alpha=+2$. If $\alpha>+2$, then the rate predictions for all surveys at frequencies $<1.4 \mathrm{GHz}$ would decrease, although there is no observational evidence arguing for $\alpha>+2$. The lower limits on the mean spectral index, $\alpha_{\text {lim }}$, are the constraints we obtain with GBNCC in the event of scattering, which are listed in Table 2.

The rate predictions for all surveys that we considered are shown in Figure 10. Our simulations predict rates that are consistent with the upper limits reported for LOFAR (Coenen et al. 2014; Karastergiou et al. 2015), AO327 (Deneva et al. 2016), MWA (Tingay et al. 2015), VLA (Law et al. 2015), and UTMOST (Caleb et al. 2016). The simulations do not account for repeating sources, and the rate reported for the PALFA survey (Scholz et al. 2016) is based on the detection of a single event. Since our simulations calculate the rate for UTMOST at its full sensitivity, the upper limit shown in Figure 10 is calculated by scaling the reported upper limit for a fluence threshold of $11 \mathrm{Jy}$ ms (Caleb et al. 2016) to the fluence for the fully sensitive UTMOST survey calculated using Equation (1).

One caveat, however, is that we have difficulty matching the predicted rate for the Parkes surveys with the observations if the mean of the scattering time distribution is set to be the observed $6.7 \mathrm{~ms}$ at $1 \mathrm{GHz}$. This suggests that one or all of the following assumptions might be incorrect: Kolmogorov spectrum, lognormal distribution of scattering timescales, width of the distribution equal to that of the Galaxy. A more sophisticated treatment of the scattering timescale distribution will allow us to make better predictions.

The results of the simulations also demonstrate the effect of the slope of the $\log N-\log S$ function on the FRB yield of a survey. The $\log N-\log S$ function determines whether field of view or sensitivity is a more important factor for FRB detection. Our simulations predict a greater FRB detection rate for PALFA as compared to Parkes for $\gamma>1$, but the rates are consistent with each other for $\gamma<1$. The abundance of fainter bursts implied by $\gamma>1$ explains the higher rate prediction for PALFA, whose greater sensitivity is highly advantageous for FRB detection in that scenario. However, if $\gamma<1$, there will be an abundance of brighter bursts, thereby allowing the greater field of view of Parkes as compared to PALFA to compensate for the reduction in sensitivity and have a similar FRB detection rate per hour.

\subsection{Predictions for CHIME}

The simulations suggest that CHIME will be detecting more FRBs than any existing telescope, due to its large field of view. However, scattering can reduce the number of detections in the lower part of the band. To model the competing effects of increase in field of view and increase in scattering timescales at lower frequencies, the CHIME bandwidth has been divided into four equal parts (centered at $450,550,650$, and $750 \mathrm{MHz}$ ) in our simulations. The scattering timescale and field of view

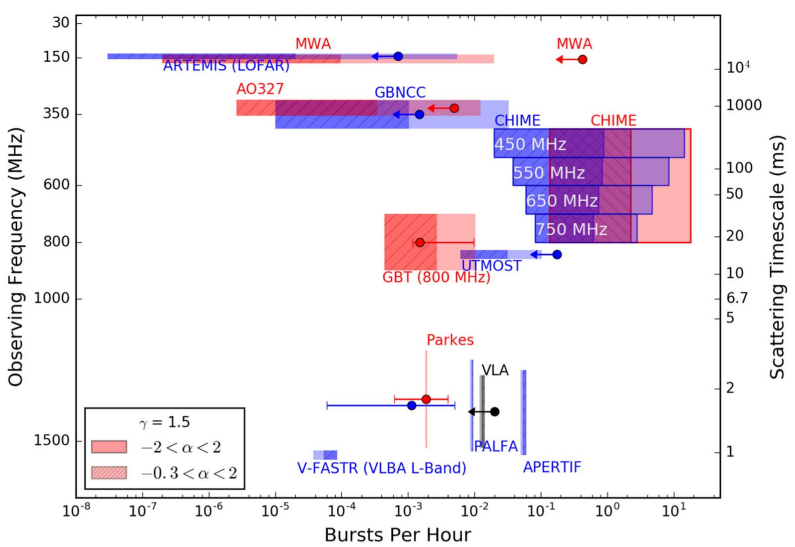

(a)

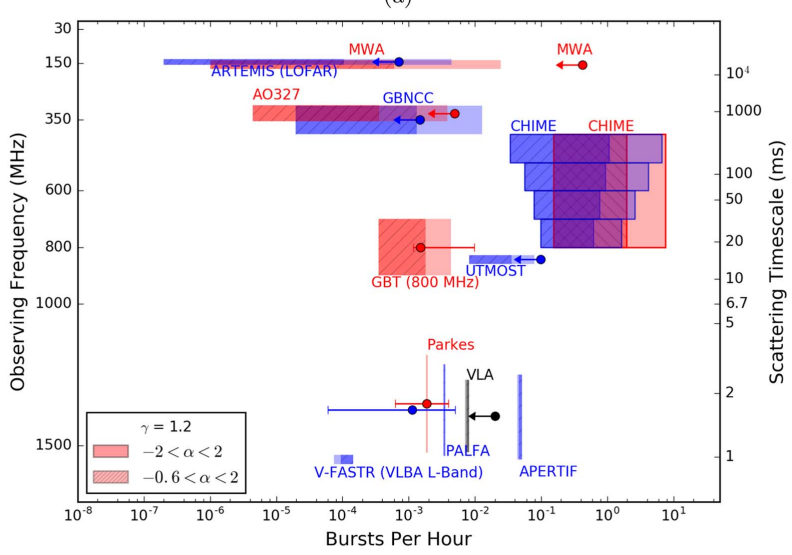

(b)

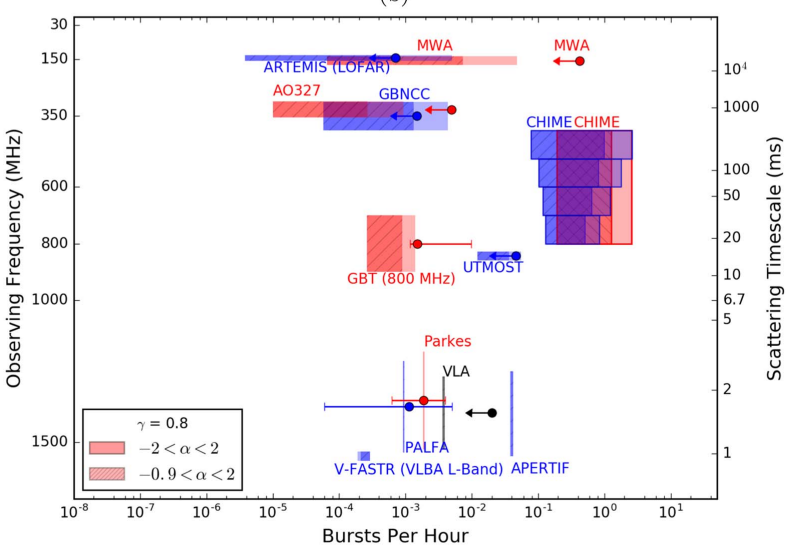

(c)

Figure 10. Predicted number of detections per hour for current and upcoming FRB surveys computed by Monte Carlo simulations using the Parkes rate reported by Crawford et al. (2016) as reference. The shaded regions correspond to the bursts per hour for an arbitrary range of spectral indices $(-2<\alpha<+2)$, while the hatched regions correspond to the bursts per hour for the range $\alpha_{\text {lim }}<\alpha<+2$, where $\alpha_{\text {lim }}$ for each $\gamma$ is the spectral index constraint derived in this paper. The shaded and hatched regions, as well as the markers denoting the published upper limits on the FRB rate (obtained from the corresponding references in Table 1), have been colored differently where there are several surveys in a certain frequency range to distinguish between the limits reported by each of these surveys. For MWA, the upper limit reported by Tingay et al. (2015) has been plotted here. Panels (a)-(c) show the predicted rates for $\gamma=1.5,1.2$, and 0.8 , respectively. The blue shaded and hatched regions in the frequency range of $400-800 \mathrm{MHz}$ represent the varying detection rates for four parts of the CHIME band with a bandwidth of $100 \mathrm{MHz}$ each. Red regions in the same frequency range represent the overall detection rate for CHIME, which has a bandwidth of $400 \mathrm{MHz}$. The regions corresponding to the four parts of the CHIME band are labeled in panel (a), with the labels denoting the center frequencies of these parts, namely, 450, 550, 650, and $750 \mathrm{MHz}$. 
for each part have been calculated using its center frequency, and the sensitivity has been evaluated using Equation (1), assuming a bandwidth of $100 \mathrm{MHz}$.

For the lower part of the CHIME band (400-500 MHz), our simulations predict $0.5-21$ bursts per day for $\gamma=1.5$ and $2-24$ bursts per day for $\gamma=0.8$. The prediction for the upper part of the CHIME band $(700-800 \mathrm{MHz})$ is $2-15$ bursts per day, assuming a Euclidean flux distribution $(\gamma=1.5)$. Connor et al. (2016) predict detection of 2-40 bursts per day for the same part of the band based on the one FRB detected with GBT at $800 \mathrm{MHz}$. Although our rate prediction for $700-800 \mathrm{MHz}$ is not very different from the Connor et al. (2016) prediction, there are significant differences in the method of rate estimation. We extrapolate the $1.4 \mathrm{GHz}$ rate estimate reported by Crawford et al. (2016) to the frequency under consideration $(750 \mathrm{MHz})$, assuming a distribution of spectral indices and scattering timescales for the FRB population. On the other hand, Connor et al. (2016) predict the detection rate based on the measured FRB rate in the relevant frequency range $(700-800 \mathrm{MHz})$ and neglect the distribution of scattering timescales. The overall rate predicted by our simulations, 3-54 bursts per day, is also in agreement with the prediction of detection of 30-100 FRBs per day by Rajwade \& Lorimer (2017) assuming a cosmological population of FRBs.

\section{Summary and Conclusions}

We did not detect any FRBs in GBNCC survey pointings amounting to a total observing time of 84 days. The nondetection allows us to determine a $95 \%$ confidence upper limit on the FRB rate at $350 \mathrm{MHz}$ of $3.6 \times 10^{3} \mathrm{FRBs} \mathrm{sky}^{-1}$ day $^{-1}$ above a peak flux density of $0.63 \mathrm{Jy}$ for bursts with an intrinsic width of $5 \mathrm{~ms}$. The threshold flux density of the survey ranges from $0.3 \mathrm{Jy}$ for an FRB of $16 \mathrm{~ms}$ duration to $9 \mathrm{Jy}$ for a $0.35 \mathrm{~ms}$ duration FRB.

We computed constraints on the mean intrinsic spectral index by performing Monte Carlo simulations of a population of FRBs consistent with the $1.4 \mathrm{GHz}$ rate estimate and assuming a power-law flux density model for FRBs. The FRBs generated in these simulations had spectral indices sampled from a normal distribution and scattering timescales sampled from a lognormal distribution. If intrinsic spectral index were the only reason for our nondetection, i.e., scattering and freefree absorption were absent, the nondetection with GBNCC would be compatible with the Parkes rate estimate reported by Crawford et al. (2016) for $\alpha>+0.35$. Karastergiou et al. (2015) derived a constraint, $\alpha>+0.1$, based on nondetection with LOFAR at $145 \mathrm{MHz}$. Nondetection with MWA at $155 \mathrm{MHz}$ implied $\alpha>-1.2$ (Tingay et al. 2015). The GBNCC survey, owing to its large observing time and greater sensitivity, thus enables us to place a stronger constraint on spectral index than has any previous survey.

However, scattering is one possible reason for our nondetection. Another variant of the simulations was aimed at finding the mean scattering timescale that would render FRBs expected for a particular value of spectral index undetectable with GBNCC. Given the observed range of scattering times at $1.4 \mathrm{GHz}$, we constrain $\alpha>-0.3$ for a Euclidean flux distribution, in the absence of free-free absorption. The constraints on spectral index are very sensitive to the $1.4 \mathrm{GHz}$ rate estimate used in the simulations. The above-mentioned constraint is derived using the Crawford et al. (2016) rate estimate. If the rate estimate reported by Champion et al. (2016) is used, then the constraint is weaker with $\alpha>-0.9$. The simulations used for deriving these constraints assume a scattering timescale distribution resembling the distribution of Earth-centered scattering times for our Galaxy. However, the scattering timescale depends on the location in, orientation of, and type of the host galaxy. Detailed treatment of this problem is beyond the scope of this paper. The simulations are also based on the assumption of a power-law spectral model. Although the assumption is in line with previous studies, it could be incorrect if the repeating FRB is a member of the same source class as the rest of the population since observations of the repeating FRB121102 (Scholz et al. 2016) show that a single power law is a poor characterization of the burst spectra.

We find that the strongest constraint is obtained for the case of the Euclidean flux distribution, both in the absence of scattering and free-free absorption and in the presence of scattering. A higher value of $\gamma$ corresponds to an increase in the relative abundance of fainter FRBs. Therefore, an increase in $\gamma$ implies an increase in the number of detections with GBNCC by virtue of its sensitivity, thereby requiring higher mean scattering times or a more positive spectral index to explain our nondetection.

For the particular case of standard candles with a constant comoving number density, we estimate a maximal redshift of 0.37 being probed by the GBNCC survey. We find a spectral index $\alpha_{\text {lim }}$ for which the peak flux density of an FRB at $z=0.37$ is equal to the survey sensitivity. We rejected any spectral index $<\alpha_{\text {lim }}$ as it would predict sensitivity to a greater redshift and hence detection of FRBs with GBNCC. In the scenario of free-free absorption with a hot ionized magnetar ejecta, we obtain $\alpha_{\text {lim }}=-0.6$, and for a cold molecular cloud having ionization fronts, we obtain $\alpha_{\text {lim }}=1.0$, under the assumption of no scattering. Our constraints imply that spectra of FRBs are different from observed pulsar spectra, for which the mean spectral index is -1.4 (Bates et al. 2013). However, if FRBs are subject to both free-free absorption and scattering, our constraints are far weaker and allow for steep negative spectral indices as well.

We also predict the detection rate for existing surveys and upcoming ones such as CHIME using Monte Carlo simulations. The simulations for a Euclidean flux distribution predict that CHIME will detect 3-54 bursts per day assuming the Crawford et al. (2016) rate estimate and 1-25 bursts per day assuming the rate estimate reported by Champion et al. (2016). The predictions are promising because even with the most conservative estimates, CHIME will be able to greatly increase the number of known FRBs and probe the distribution of their properties such as spectral index, scattering timescales, and the slope of the $\log N-\log S$ function.

The National Radio Astronomy Observatory is a facility of the National Science Foundation operated under cooperative agreement by Associated Universities, Inc. We thank Compute Canada, the McGill Center for High Performance Computing, and Calcul Quebec for provision and maintenance of the Guillimin supercomputer and related resources. We also thank an anonymous referee for useful comments that helped improve the manuscript. We are grateful to Erik Madsen for providing code to make plots for Figure 10. P.C. acknowledges support from a Mitacs Globalink Graduate Fellowship and the TOEFL Scholarship Program in India. V.M.K. receives support from 
an NSERC Discovery Grant, an Accelerator Supplement, a Gerhard Herzberg Award, an R. Howard Webster Foundation Fellowship from the Canadian Institute for Advanced Research, the Canada Research Chairs Program, and the Lorne Trottier Chair in Astrophysics and Cosmology. J.W.T.H. and V.I.K. acknowledge support from the European Research Council under the European Union's Seventh Framework Programme (FP/2007-2013)/ERC Grant Agreement no. 337062. MAM is supported by NSF AST Award no. 1211701. Pulsar research at UBC is supported by an NSERC Discovery Grant and by the Canadian Institute for Advanced Research. J.v.L. acknowledges funding from the European Research Council under the European Union's Seventh Framework Programme (FP/2007-2013)/ERC Grant Agreement no. 617199.

Software: PRESTO (Ransom 2001), RRATtrap (KarakoArgaman et al. 2015).

\section{ORCID}

P. Chawla (iD https://orcid.org/0000-0002-3426-7606

V. M. Kaspi (D) https://orcid.org/0000-0001-9345-0307

D. R. Lorimer (iD https://orcid.org/0000-0003-1301-966X

A. M. Archibald (iD https://orcid.org/0000-0003-0638-3340

M. E. DeCesar (ib https://orcid.org/0000-0002-2185-1790

J. W. T. Hessels (i) https://orcid.org/0000-0003-2317-1446

D. L. Kaplan (iD https://orcid.org/0000-0001-6295-2881

L. Levin (i) https://orcid.org/0000-0002-2034-2986

R. S. Lynch (1) https://orcid.org/0000-0001-5229-7430

M. A. McLaughlin (i) https://orcid.org/0000-0001-7697-7422

S. M. Ransom (ib https://orcid.org/0000-0001-5799-9714

M. S. E. Roberts (i) https://orcid.org/0000-0002-9396-9720

I. H. Stairs (1) https://orcid.org/0000-0001-9784-8670

K. Stovall (iD https://orcid.org/0000-0002-7261-594X

J. K. Swiggum (1) https://orcid.org/0000-0002-1075-3837

\section{References}

Ade, P. A. R., Aghanim, N., Arnaud, M., et al. 2016, A\&A, 594, A13 Anderberg, M. R. 1973, Cluster Analysis for Applications (New York: Academic)

Bannister, K. W., \& Madsen, G. J. 2014, MNRAS, 440, 353

Bates, S. D., Lorimer, D. R., \& Verbiest, J. P. W. 2013, MNRAS, 431, 1352

Bentley, J. L. 1975, Communications of the ACM, 18, 509

Burke-Spolaor, S., \& Bannister, K. W. 2014, ApJ, 792, 19

Burke-Spolaor, S., Trott, C. M., Brisken, W. F., et al. 2016, ApJ, 826, 223

Caleb, M., Flynn, C., Bailes, M., et al. 2016, MNRAS, 458, 718

Champion, D. J., Petroff, E., Kramer, M., et al. 2016, MNRAS, 460, L30

Chatterjee, S., Law, C. J., Wharton, R. S., et al. 2017, Natur, 541, 58

Coenen, T., van Leeuwen, J., Hessels, J. W. T., et al. 2014, A\&A, 570, A60

Connor, L., Lin, H.-H., Masui, K., et al. 2016, MNRAS, 460, 1054
Cordes, J. M., \& Lazio, T. J. W. 2002, arXiv:astro-ph/0207156

Cordes, J. M., \& McLaughlin, M. A. 2003, ApJ, 596, 1142

Cordes, J. M., \& Wasserman, I. 2016, MNRAS, 457, 232

Cordes, J. M., Wharton, R. S., Spitler, L. G., et al. 2016, arXiv: 1605.05890

Crawford, F., Rane, A., Tran, L., et al. 2016, MNRAS, 460, 3370

Deneva, J. S., Stovall, K., McLaughlin, M. A., et al. 2016, ApJ, 821, 10

Ester, M., Kriegel, H.-P., Sander, J., \& Xu, X. 1996, in Proc. 2nd Int. Conf. on Knowledge Discovery and Data Mining, ed. E. Simoudis, J. Han, \& U. Fayyad (Portland, OR: AAAI Press), 226

Haslam, C. G. T., Salter, C. J., Stoffel, H., \& Wilson, W. E. 1982, A\&AS, 47, 1 Inoue, S. 2004, MNRAS, 348, 999

Ioka, K. 2003, ApJL, 598, L79

Karako-Argaman, C., Kaspi, V. M., Lynch, R. S., et al. 2015, ApJ, 809, 67

Karastergiou, A., Chennamangalam, J., Armour, W., et al. 2015, MNRAS, 452,1254

Katz, J. I. 2016, MPLA, 31, 1630013

Keane, E. F., Johnston, S., Bhandari, S., et al. 2016, Natur, 530, 453

Keane, E. F., Stappers, B. W., Kramer, M., \& Lyne, A. G. 2012, MNRAS, 425, L71

Kulkarni, S. R., Ofek, E. O., \& Neill, J. D. 2015, arXiv:1511.09137

Law, C. J., Bower, G. C., Burke-Spolaor, S., et al. 2015, ApJ, 807, 16

Lorimer, D. R., Bailes, M., McLaughlin, M. A., et al. 2007, Sci, 318, 777

Lorimer, D. R., Karastergiou, A., McLaughlin, M. A., \& Johnston, S. 2013, MNRAS, 436, L5

Lorimer, D. R., \& Kramer, M. 2005, Handbook of Pulsar Astronomy (Cambridge: Cambridge Univ. Press)

Macquart, J.-P., \& Johnston, S. 2015, MNRAS, 451, 3278

Masui, K., Lin, H.-H., Seivers, J., et al. 2015, Natur, 528, 523

Mezger, P. G., \& Henderson, A. P. 1967, ApJ, 147, 471

Oppermann, N., Connor, L. D., \& Pen, U. 2016, MNRAS, 461, 984

Petroff, E., Bailes, M., Barr, E. D., et al. 2015, MNRAS, 447, 246

Petroff, E., Barr, E. D., Jameson, A., et al. 2016, PASA, 33, 45

Petroff, E., van Straten, W., \& Johnston, S. 2014, ApJL, 789, L26

Popov, S. B., \& Postnov, K. A. 2013, arXiv:1307.4924

Rajwade, K. M., \& Lorimer, D. R. 2017, MNRAS, 465, 2286

Ransom, S. M. 2001, PhD thesis, Harvard Univ.

Ravi, V., Shannon, R. M., Bailes, M., et al. 2016, Sci, 354, 1249

Ravi, V., Shannon, R. M., \& Jameson, A. 2015, ApJL, 799, L5

Remazeilles, M., Dickinson, C., Banday, A. J., et al. 2015, MNRAS, 451, 4311

Rowlinson, A., Bell, M. E., Murphy, T., et al. 2016, MNRAS, 458, 3506

Scholz, P., Spitler, L. G., Hessels, J. W. T., et al. 2016, ApJ, 833, 177

Spitler, L. G., Cordes, J. M., Hessels, J. W. T., et al. 2014, ApJ, 790, 101

Spitler, L. G., Scholz, P., Hessels, J. W. T., et al. 2016, Natur, 531, 202

Stovall, K., Lynch, R. S., Ransom, S. M., et al. 2014, ApJ, 791, 67

Tendulkar, S. P., Bassa, C. G., Cordes, J. M., et al. 2017, ApJL, 834, 2

Thornton, D., Stappers, B., Bailes, M., et al. 2013, Sci, 341, 53

Tingay, S. J., Goeke, R., Bowman, J. D., et al. 2013, PASA, 30, 7

Tingay, S. J., Trott, C. M., Wayth, R. B., et al. 2015, AJ, 150, 199

van Leeuwen, J. 2014, in Proc. The Third Hot-wiring the Transient Universe Workshop, ed. P. R. Wozniak et al. (Stanford, CA: Stanford Univ. Press), 79, http://www.slac.stanford.edu/econf/C131113.1/

Vander Wiel, S., Burke-Spolaor, S., Lawrence, E., et al. 2016, arXiv:1612. 00896

Vedantham, H. K., Ravi, V., Hallinan, G., et al. 2016a, ApJ, 830, 75

Vedantham, H. K., Ravi, V., Mooley, K., et al. 2016b, ApJL, 824, L9

Wayth, R. B., Tingay, S. J., Deller, A. T., et al. 2012, ApJL, 753, 2

Williams, P. K. G., \& Berger, E. 2016, ApJL, 821, L22 